\title{
POSITIVISMO GIURIDICO E STUDIO DEL DIRITTO COSTITUZIONALE*
}

\author{
Francesco Bilancia
}

\begin{abstract}
ASTRATTO

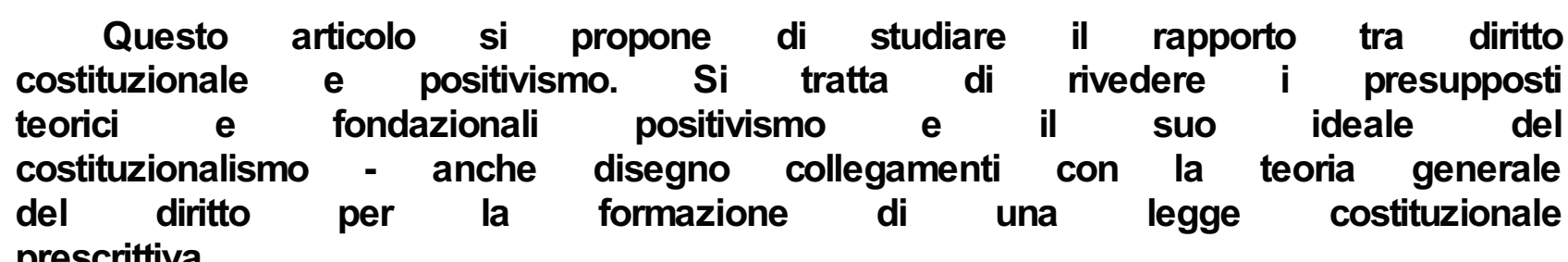

Parole chiave: Diritto costituzionale. Diritto costituzionale prescrittiva. Positivismo. Teoria generale del diritto.

\section{UNA PREMESSA: LA QUESTIONE DEI “PUNTI DI VISTA”}

Nella sterminata produzione scientifica dedicata da Alessandro Pace ai più svariati ed importanti temi del diritto costituzionale gli scritti in cui è presente anche solo qualche riflessione intorno all'approccio di metodo consapevolmente seguito dall'illustre studioso sono essi stessi molto numerosi. Ma in alcuni più recenti, importanti, lavori le riflessioni sul metodo professato e, più in generale, sulla interpretazione costituzionale diventano ancora più meditate, tanto da apparire centrali nella qualificazione delle stesse linee di sviluppo delle ricerche condotte ${ }^{1}$.

La chiarezza argomentativa e la consapevole esposizione dei presupposti di metodo che guidano la riflessione dello scienziato sociale, avvenga o meno quest'ultima in una esplicita declaratoria, dovrebbero rendere infatti giustizia di molti dei corollari del punto di vista culturale e scientifico a fondamento dei propri ragionamenti, così contribuendo a risolvere pregiudizialmente equivoci e semplificazioni che ancora di recente si sono spesso rivelati causa di incomprensioni - non saprei dire se vere 0 soltanto polemicamente insistite - e di acceso confronto ${ }^{2}$. Come vedremo subito, però, ogni polemica presa di posizione sul metodo - proprio o altrui - non pare potersi affievolire sul solo presupposto di avere preventivamente declinato il punto di vista assunto a fondamento della propria riflessione ${ }^{3}$. Forse, allora, nel campo degli studi giuridici il punto di vista degli studiosi andrebbe a volte collocato esso stesso ad oggetto della riflessione scientifica assumendolo nelle premesse dei propri ragionamenti come vera e propria categoria critica. E' ciò che appunto è accaduto in relazione al confronto costruito nelle relative inferenze di presupposti e di metodo tra positivisti e giusnaturalisti, le cui reciproche qualificazioni critiche hanno da sempre costituito oggetto di studio e di importanti riflessioni ${ }^{4}$.

Analogamente ciò dovrebbe verificarsi nel costante reciproco confronto tra giuristi "positivi" e filosofi del diritto ${ }^{5}$, supponendo di poter distinguere a priorie schematicamente oggetto di studio e metodo di lavoro degli uni e degli altri, ammettendo altresì che sia possibile costruire sedi e modi di oggettiva classificazione degli studiosi nella presunta 
appartenenza all'uno o all'altro ambito degli studi, selezionando e valutando poi a tal fine gli elementi della riflessione ed i relativi ambiti materiali di ricerca declinati, come dire?, per sedi separate. Come è evidente la cosa non solo è impossibile ma forse anche inutile e banale, anzi inutile in quanto banalizzante questioni e tematiche la cui elaborazione schematica porterebbe ad una deriva semplificatrice, probabilmente neanche passibile di essere inquadrata in un metodo qualificabile come scientifico. Questa premessa parte dal presupposto, intuito più che dimostrato, che la ricognizione ed il confronto tra i diversi punti di vista degli studiosi siano essi stessi il prodotto di una lettura orientata da presupposti culturali e di metodo - quelli dell'interprete del pensiero altrui - che, come tali, non possono assumere valenza neutra ed oggettiva. Ogni riflessione sul tema apparendo, pertanto, essa stessa plausibile di osservazione oggettiva dall'esterno, concorrendo alla costruzione di un ovvio anche se consapevole circolo ermeneutico tra oggetto e metodo di osservazione e valutazione della riflessione altrui, ma così rendendo forse impossibile l'analisi dei differenti punti di vista ${ }^{6}$ a prescindere, appunto, dal proprio.

Aggravato forse, il proprio punto di vista, da una presa di posizione che si pretenda metateorica. Diamo per scontato già in premessa allora che ogni qualificazione a fondamento della riflessione sul positivismo si assume, pertanto, come implicante il punto di vista dell'autore, suscettibile di osservazione esso stesso salve le opportune chiarificazioni stipulative che, almeno in via di metodo, si ritiene ora di poter formulare.

II punto di partenza si connette ad una primitiva intuizione, prodotto della riflessione sui diversi studi alla base di queste note, messi a confronto con l'intento di isolarne fin da subito i presupposti di metodo professati o comunque impliciti nella impostazione prescelta dai diversi autori e dando per scontato che quest'ultima sia il prodotto di un'assunzione consapevole. Un errore nella individuazione e nella valutazione di tale premessa è, pertanto, implicitamente possibile e renderebbe, allora, necessario rimettere in discussione ogni volta le nostre conclusioni, in un continuo proficuo confronto - appunto dei differenti punti di vista per la loro reciproca (ma non solo, anche presuntivamente oggettiva) definizione.

Per cominciare direi, pertanto, che i giuristi ed i filosofi del diritto ${ }^{7}$ osservano oggetti different $^{\beta}$, cosa che non dovrebbe necessariamente condurre ad una confusione dei linguaggi né ad un problema di convergenza terminologica nei rispettivi impianti culturali a confronto". Allorché, quindi, ci si accinga ad avviare una riflessione sul "positivismo giuridico,10 e sull'influenza che nello studio di tale oggetto venga esercitata dall'ideologia giuridica $^{11}$ professata dai diversi studiosi, preliminare sarebbe tentare di dare risposta alla domanda: ma quale diritto positivo osserviamo?

\section{POSITIVISMO GIURIDICO E DIRITTO POSITIVO}

II più delle volte non è semplice assumere questo interrogativo come base di partenza della discussione, per la difficoltà di dare ad esso risposta sia in termini assoluti - quale diritto positivo è effettivamente oggetto di osservazione da parte di ciascuno studioso? - sia in termini relativi, procedendo al confronto dell'oggetto e del metodo di ricerca di ciascuno studioso. Semplificando un po' il percorso di analisi è, però, forse possibile cogliere intanto alcuni elementi intuitivi che potrebbero, proseguendo nella riflessione, concorrere a fare chiarezza almeno delle differenze di piano in cui si collocano effettivamente gli oggetti dello studio di filosofi del diritto e del linguaggio e dei giuristi (positivi $^{12}$. Con ciò riprendendo, ma solo in parte, l'osservazione formulata da Hart ${ }^{13}$ circa $^{2}$ 
l'attitudine dei giuristi ad interrogarsi sul proprio oggetto di studio ${ }^{14}$. Solo in parte perché qui la riflessione coinvolge quale proprio oggetto non solo - o non tanto - il diritto ma lo stesso punto di vista degli studiosi. Mi verrebbe, così, da concludere fin da subito con una domanda retorica: ma i giuristi che ritengono di riconoscersi in una delle molte versioni contemporanee del c.d. "positivismo giuridico"15, che verosimilmente si ascriverebbero nel novero dei positivisti, davvero osservano e studiano il diritto positivo? $\mathrm{E}$, se la risposta fosse affermativa, a quale diritto positivo essi fanno riferimento? Al diritto vigente di quale ordinamento giuridico positivo volgono la propria attenzione ${ }^{16}$ ? Insomma, i positivisti davvero osservano e studiano il diritto positivo?

Questa domanda, nei diversi corollari in cui è stata qui articolata, è la dimostrazione intuitiva della rilevanza dei punti di vista e della rilevazione consapevole della presenza di un pesante diaframma di incomunicabilità tra i diversi approcci. Denunciato il quale è, pertanto, possibile tornare al nostro punto di vista, per elaborare una riflessione nell'ottica del costituzionalista, insomma di uno studioso del diritto costituzionale positivo contemporaneo al netto delle diversità di approccio di metodo pur compresenti nel panorama degli studiosi della disciplina ${ }^{17}$.

Così a proposito dei punti di osservazione, non sembra inutile notare da subito che tra gli oggetti della propria riflessione, ancorché non sempre e non da parte di tutti, ma certo più spesso rispetto agli studiosi delle altre discipline, i costituzionalisti pongono il problema dei fondamenti del fenomeno giuridico, la questione cioè dei rapporti tra il potere ed il diritto. Carl Schmitt, come è noto, discorreva del succedersi nel tempo e nella storia di successivi processi di neutralizzazione dei centri di riferimento culturale ${ }^{18}$, ma oltre il susseguirsi delle varie epoche così descritte il problema dei rapporti tra diritto e politica rimane immanente al fenomeno giuridico, più che mai nell'epoca contemporanea.

Nello studio del diritto costituzionale, inoltre, procedendo anche soltanto ed inizialmente con mero approccio descrittivo ${ }^{19}$ i costituzionalisti non possono non essere necessariamente condizionati, nelle loro indagini, dal modo di essere della Costituzione, dalla sua natura di documento politico e dalla sua struttura normativa, non più fedele ormai ai paradigmi formali del positivismo legalistico ottocentesco ${ }^{20}$. I documenti costituzionali attuali, oggetto di osservazione degli studiosi del diritto costituzionale - in quanto diritto positivo vigente - selezionano infatti, dando ad essi piena valenza prescrittiva, anche i fini dell'ordinamento, qualificabili come il dover essere del diritto positivo di matrice legislativa ${ }^{21}$. Questi fini sono, appunto, posti dalla Costituzione spesso nella forma di principi (o valori) ${ }^{22}$, tipi di norme giuridiche nei cui confronti gli studiosi di diritto costituzionale non possono non assumere un atteggiamento valutativo ${ }^{23}$, utilizzandoli come parametro di giudizio nell'osservazione del farsi dell'ordinamento medesimo.

Da quanto fin qui osservato è già forse possibile assumere, quale premessa di metodo, un dato giuspolitico che si ritiene valido in quanto oggettivamente osservabile nel tempo e nello spazio, insomma, nella storia: dietro ogni sistema giuridico ${ }^{24}$ si cela sempre un principio politico normativizzato ${ }^{25}$, nel senso di positivizzato, che ne costituisce il fondamento, sul presupposto che la produzione del diritto, anche laddove si svolga nel rispetto delle norme sulla produzione vigenti in quel dato momento, nel rispetto cioè del diritto positivo vigente in quello specifico ordinamento, esprime comunque la concreta relazione storica della politica con il diritto ${ }^{26}$. Così l'ordinamento italiano costituito dalla Carta del 1948 compone il suo sistema giuridico articolando le fonti del diritto secondo i principi della democrazia costituzionale; un ordinamento fondato sulla sovranità popolare 
esercitata "nelle forme e nei limiti della Costituzione", articolata perciò secondo il modello della democrazia rappresentativa nel quadro dei limiti definiti da una Costituzione rigida e garantita come "legge superiore".

Questa ulteriore constatazione potrà così fungere da premessa in concreto, svolgimento delle cautele formulate in apertura, per riflettere sugli oggetti della osservazione del giurista studioso del diritto positivo vigente, più nello specifico del costituzionalista.

Se non può non assumersi quale punto di partenza la primitiva constatazione che il diritto si presenta, essenzialmente, come insieme di regole, come complesso oggettivo di norme, il problema che pone la specifica analisi del costituzionalista, secondo il suo proprio statuto disciplinare, sarà innanzi tutto quello di procedere ad una valutazione del proprio oggetto di studio al necessario fine di ricondurlo al suo proprio fondamento, vale a dire alla sua fonte, alla sua origine, al suo proprio autore. E' il problema, cioè, di rispondere alla domanda: perché dobbiamo obbedire a queste regole? Qual è la giustificazione giuspolitica della giuridicità di questo sistema, la causa della obbligatorietà dei suoi elementi normativi? II diritto è, inoltre, un fenomeno propriamente sociale, perviene cioè all'organizzazione di una determinata società. Questo insieme di regole rappresenta, in sostanza, il prodotto di una società organizzata, ed ha per fine la disciplina, l'ordinamento della società medesima e dei rapporti tra i membri di questa. Questa esigenza conduce alla necessità di provvedere allo stabilizzarsi di regole che, per quanto rudimentali, consentano ai membri di convivere in pace tra loro. Sarà la forza bruta o il carisma del capo, la convenienza per motivi di difesa del gruppo medesimo, o l'amore tra i suoi membri, ma alla fine quell'insieme di soggetti si organizzerà attraverso regole, costituendosi in fenomeno sociale ordinato, disciplinato cioè mediante diritto.

II fenomeno giuridico viene così ad essere valutato in base alla propria funzione ${ }^{27}$, ma nulla esclude che si possa procedere altrimenti nel definire la sua stessa propria natura. II diritto ad esempio è più spesso valutato in relazione alla sua struttura, alla sua forma; oppure in relazione ai contenuti di valore cui si ispira e che intende realizzare o, infine, in base alla volontà del potere, dell'autorità dalle cui decisioni provengono i comandi di cui si compone. In questa breve introduzione siamo già riusciti ad individuare almeno quattro differenti profili del fenomeno giuridico cui possono corrispondere metodi altrettanto distinti di rappresentazione della sua propria essenza, approcci e modelli di osservazione e valutazione del diritto che storicamente la scienza ha altresì a volte composto tra loro in differenti combinazioni 0 , altrimenti, gerarchicamente ordinato ${ }^{28}$. Questa esemplificazione ci riporta così al rilievo dei presupposti storico-politici più sopra richiamato. II diritto è sempre riconducibile ad un sistema di valutazione, ad un paradigma composto quale insieme di opzioni politiche che nel rappresentarne storicamente il fondamento concorrono altresì a qualificare l'osservazione, lo studio e la riflessione che su tale oggetto vengano condotte ${ }^{29}$. II motivo storico-politico per il quale in un determinato momento ed in un particolare luogo si reputi normativamente giusto, o doveroso, rispettare determinate regole giuridiche, cioè, si fonda sempre su una specifica scelta di metodo, in base alla quale viene poi definito il criterio culturale chiamato a rappresentare, nella riflessione scientifica, il fondamento stesso del diritto. Giusto, naturalmente, in un'ottica giuridica, con riguardo cioè alla effettiva obbligatorietà delle norme, non nell'ottica della morale, propria del c.d. 'formalismo etico". L'interrogativo qui si rivolge alla obbligatorietà del diritto in quanto causa della sua stessa positività, non invece con riguardo all'atteggiamento interiore dell'interprete. 
Così continuando nell'esempio il diritto può essere considerato, ed è stato storicamente considerato, a) come l'insieme dei comandi e delle regole di provenienza da una determinata autorità politica. II diritto, cioè, è stato inteso come "ciò che piace al sovrano", sia esso un tiranno, un'assemblea rivoluzionaria, un interprete della volontà divina rivelata, la volontà dei Padri, cioè, nel tempo, la tradizione; fino a considerare diritto quell'insieme di regole prodotte da un organo a ciò appositamente deputato, come ad esempio un Parlamento. A ben vedere, infatti, il fenomeno giuridico, la sua obbligatorietà, la coercibilità delle sue regole, vale a dire la concreta possibilità materiale - non astratta e concettuale, quindi, ma storicamente reale - che il rispetto di tali regole venga imposto, se necessario, con la forza, è direttamente connesso al potere politico dominante. Potere inteso come fonte del diritto, da un lato, considerando allo stesso tempo quest'ultimo come strumento del potere. II sovrano, cioè, agisce e si manifesta attraverso regole giuridiche di cui impone l'osservanza con la forza. E come sua manifestazione, dall'altro, laddove il diritto venga considerato unica fonte legittima del potere. II sovrano è esso stesso, in questa prospettiva, tenuto a rispettare le regole giuridiche, al di fuori delle quali il suo potere è valutato come arbitrario e, per ciò stesso, illegittimo. In questa prima prospettiva non interessa la forma in cui il diritto si manifesti, né la qualità, in termini di giustizia, dei suoi contenuti. L'idea di conferire ad un determinato organo, secondo uno specifico procedimento, la capacità, meglio, la funzione di produrre diritto, conduce invece al b) concetto di forma. Diritto è, pertanto, soltanto la regola che appaia prodotta secondo determinate regole, da un determinato organo in base alle prescritte procedure e che si presenti in una determinata veste formale. Mentre la configurazione del diritto come fonte, e quindi come limite, del potere, da altra prospettiva fa da premessa all'idea, anch'essa come le precedenti politicamente orientata, c) che il diritto sia riconoscibile per via dei suoi contenuti di valore, che il potere sia legittimo soltanto se conforme al diritto e che, per l'appunto, diritto (giusto) sia soltanto quello che abbia, ad esempio - secondo i canoni della civiltà giuridica contemporanea - a proprio contenuto la pretesa di limitare il potere. Così è, come è noto, per la dottrina del costituzionalismo.

Nell'approccio storico-politico indicato alla lettera a) molti studiosi identificano la matrice storica del positivismo legalistico ottocentesco nelle sue diverse versioni, con particolare riferimento al modello incarnato dallo statualismo tedesco ${ }^{30}$. Secondo tale approccio "la validità "positiva" della legge è necessariamente sempre...qualcosa di fattuale", ma appunto perciò "il positivista sarà naturalmente portato ad intendere il problema dell'inizio della validità positiva della norma come un problema non più giuridico,31. A partire da Laband è infatti iniziato il lungo percorso di depurazione dello studio del diritto - in particolare del diritto pubblico - da interferenze della filosofia, della politica e dell'ideologia per assumere una propria autonoma configurazione dogmatica, mediante una costruzione funzionale però alla elaborazione dello statalismo volontarista ${ }^{32}$ di Bismarck.

Guardando, invece, alla forma essa dà innanzi tutto rilievo alla configurazione esteriore degli atti che contengono le regole di diritto. Secondo questa prospettiva di metodo il diritto si qualifica in quanto prodotto di un procedimento previamente definito da norme giuridiche, che si presenti connotato secondo precisi caratteri esteriori, formali. Ma questa prospettiva di metodo si astrae ulteriormente dalla concretezza del farsi del diritto in seno ai sistemi politico-sociali e tende a risolversi altresì in una pretesa configurazione della struttura del sistema giuridico e delle sue norme ${ }^{33}$, predicandone appunto una specifica e necessaria connotazione strutturale ${ }^{34}$. Da questa impostazione discenderebbe, così, l'impegno a sottoporre all'impero delle regole non tanto - non solo il sistema sociale ed i suoi conflitti, ma la stessa teoria del diritto, in una professione di 
metodo che declini il formalismo positivista come una scienza pratica, in un quadro di riferimento prescrittivo per lo stesso sviluppo dell'approccio culturale e di metodo dello studioso ${ }^{35}$.

Ma se l'interesse dello studioso - così è in genere per il costituzionalista - è piuttosto orientato alla osservazione in concreto di un reale sistema positivo, storicamente collocato e determinato, la scelta del criterio di orientamento per la comprensione del fenomeno giuridico finisce con l'essere anch'essa conseguentemente condizionata dalla individuazione e dalla valutazione critica della stessa ideologia politico-culturale che storicamente ne rappresenti la matrice, dovendo riconoscere nel diritto il prodotto di tale cultura. La derivazione del diritto positivo da un impianto giuspolitico volontarista; la rilevanza della qualità strutturale del materiale giuridico, vale a dire la centralità della forma quale carattere identificativo e distintivo del diritto, matrice della stessa validità delle norme; o ancora il dominio esercitato dai contenuti di valore professati dall'ordinamento si alternano infatti storicamente quali caratteri della giuridicità a seconda della forma di stato che si affermi nella realtà politica di riferimento.

L'approccio storicista $^{36}$ consente, infatti, di comprendere quale oggetto di osservazione anche la crisi politica del sovrano, vale a dire del sistema politico dominante, che trascina con sé la perdita di effettività delle fonti del diritto che di quel sistema, di quelle autorità politiche siano stata espressione. Così è avvenuto, ad esempio, per i poteri normativi del re, o dell'imperatore, mano a mano che si è venuta affermando l'autorità politica del Parlamento, che ha fatto sì che la legge si imponesse nel sistema a scapito dei decreti reali e delle ordinanze imperiali. In altro contesto culturale, di lì a poco, sarà, piuttosto la forma a diventare il carattere distintivo del diritto, qualificandone il valore in termini di neutralità dei contenuti ideali, filosofici, religiosi o politici, nei confronti dei quali il sistema pretenderà di professare una prescrittiva indifferenza. E' la crisi dello stato liberale borghese ad aver mutato il ruolo della legge - e quindi del diritto - atto normativo divenuto fondamentale non più in quanto atto rappresentativo della classe sociale dominante, ma perché espressione della volontà generale rappresentata in Parlamento. La legge diverrà, cioè, strumento di normazione, neutrale rispetto agli interessi in conflitto, in quanto mera forma di regolazione, disciplinata in un procedimento aperto a tutti gli interessi legittimamente rappresentati, ma equidistante da ciascuno di essi, ed orientata al bene comune, all'imparzialità, all'eguaglianza. Da qui l'acquisizione della centralità della forma, vale a dire della neutralità delle procedure, divenute l'elemento distintivo della giuridicità come suo carattere essenziale; da qui la valenza garantista del paradigma formale e l'assunzione del formalismo quale carattere proprio della specificità del giuridico ${ }^{37}$.

Almeno fino all'avvento delle costituzioni rigide ${ }^{38}$. Allorché la neutralità della legge sarà giuridicamente costretta a misurarsi con i valori positivi propugnati dalla legge superiore, cioè con i programmi previsti dalla costituzione, assumendo il compito di realizzarne gli obiettivi, e privilegiando determinati fini ed interessi incarnati dalla forma di stato dominante, la democrazia partecipativa e la giustizia sociale ${ }^{39}$. Con ciò muteranno nuovamente i presupposti teorici su cui fondare la definizione del fenomeno giuridico ${ }^{40}$. Così, quasi a voler anticipare le nostre conclusioni, pare indiscutibile la necessità di riformulare il quadro metodico della riflessione scientifica relativa allo studio del diritto costituzionale attuale - starei per dire del diritto costituzionale positivo - per procedere nel senso della sintesi tra le prospettive culturali più sopra individuate nelle formule descritte nei punti b) e c), assumendo la centralità della forma ma senza pretermettere la rilevanza dei contenuti di valore quali caratteri propri della giuridicità positiva ${ }^{41}$. Di questa 
fanno, infatti, parte anche le scelte politiche normativizzate, vale a dire positivizzate negli attuali documenti costituzionali. Valori e fini prescritti dalla costituzione e declinati secondo le forme indicate dagli stessi testi costituzionali; vale a dire fini articolati in principi e valori al cui perseguimento si orientino gli atti normativi adottati nel rispetto delle forme legali definite dalla costituzione medesima.

Elementi normativi questi che impongono ancora di leggere ed interpretare i testi costituzionali secondo il metodo "uclassico» (testuale-sistematico) di origine giuspositivista (...) ma attento agli sviluppi della storia",A2.

Quindi i diversi punti di vista molti e differenti, insomma plurimi, andrebbero tutti volta a volta giustificati e confrontati sulla base delle caratteristiche giuspolitiche dello specifico diritto positivo oggetto di osservazione. Date infatti le sue stesse premesse teoriche non può esistere un solo positivismo giuridico, buono per ogni regime politico, o giuridico che dir si voglia, che l'esperienza storica produca di volta in volta e nei diversi luoghi e sistemi ordinamentali. A meno di non voler seguire prospettive di riflessione orientate, come accennato in premessa, su oggetti di studio differenti dal diritto positivo vigente in un determinato contesto storico (e territoriale?), tenuto conto che molti aderenti al movimento del positivismo giuridico - a volte in alcune delle sue declinazioni pratiche qualificato come «neopositivismo» - non sono, appunto, studiosi di diritto positivo.

Lasciando quindi per il momento senza risposta la domanda 'Che cos'è il positivismo giuridico?'43 proverò a riflettere sulla differente questione "Qual è...", oppure "Come è....", oppure “Come si presenta...il diritto positivo?", al fine di rendere evidente il presupposto essenziale del mio approccio, del mio punto di vista. Ciò anche a costo delle forti approssimazioni dovute alla relativizzazione della stessa idea del diritto in sé, al fine di non mettere già ora in discussione la natura stessa del fenomeno giuridico.

\section{POSITIVISMO E COSTITUZIONALISMO}

Le premesse formulate nel precedente paragrafo sulla nozione stessa di positivismo giuridico suggeriscono pertanto di chiarire perché il costituzionalismo, a far data dalla sua maturazione consapevole, si sia posto storicamente in conflitto con il positivismo legalistico ottocentesco ${ }^{44}$, ciò anche al fine di ridurre l'alto tasso di incomprensione manifestatosi a volte nel dialogo tra alcuni dei costituzionalisti e dei filosofi del diritto ${ }^{45}$.

Così i riferimenti ai difetti, per non dire ai "crimini" del positivismo giuridico quale strumento dell'autoritarismo, che di recente hanno riaperto la discussione polemica sull'argomento a seguito del citato saggio di Antonio Baldassarre ${ }^{46}$, sono con tutta evidenza da imputare alla versione del positivismo storico ottocentesco come già più sopra ricordato, e non certo $^{47}$ alle attuali declinazioni filosofico-giuridiche della dottrina kelseniana del diritto. E' la relazione tra positivismo storico e statalismo ad aver generato, come è noto, i dubbi e le critiche su questa concezione del diritto e dell'ordinamento giuridico. La ragione di tale approccio polemico è, allora, da ricercare paradossalmente proprio nell'attitudine degli studiosi ad osservare il diritto positivo senza giustificarlo 0 , addirittura e forse peggio, valutarlo. II metodo scientifico propriamente detto impone invece - ha imposto - di identificare in ogni epoca- così nella fase delle origini e del primo sviluppo del positivismo storico - natura e fondamento giuspolitico del diritto e dell'ordinamento effettivamente vigente nel tempo e nello spazio osservato, indicandone conseguentemente l'effettivo carattere sostanziale. E' così che questo paradigma viene 
oggi applicato nel disvelamento dei fondamenti culturali, politici e scientifici dei modelli a suo tempo elaborati da Paul Laband in funzione di Bismarck per il diritto positivo tedesco 48 ; e da Vittorio Emanuele Orlando con l'invenzione del "metodo giuridico", in funzione della unità ed identità del diritto statale italiano ${ }^{49}$. In entrambi i casi la scienza giuridica ufficiale si è venuta declinando espressamente in funzione legittimante l'esistente giuridico costruito (o in costruzione) ad opera del nuovo regime politico statuale in formazione, giustificando perciò una conseguente riflessione critica sul reale senso dell'autonomia della scienza del diritto pubblico e sull'effettiva origine storica della teorica del formalismo ${ }^{50}$.

Per chiarire meglio questo assunto ritengo utile, allora, fare riferimento ad una concreta esperienza storica al fine di dimostrare la necessità, a volte, di scindere tra loro il positivismo giuridico dal formalismo logicista e dallo stesso normativismo laddove si intenda osservare, analizzare e comprendere ogni specifica versione storica di positivismo senza con ciò voler intaccare la coerenza sistemica e la correttezza di metodo delle teoriche giuspositiviste contemporanee.

Quale esempio paradigmatico del significato critico della nozione di positivismo nel senso descritto mi sembra, così, assai indicativo il giudizio espresso su Carl Schmitt in un bellissimo saggio dedicatogli da Alberto Predieri ${ }^{51}$, nel quale l'autore tedesco, apparentemente rovesciando il significato del paradigma, "per contrappasso, è annoverato (...) tra i campioni del positivismo in senso giuridico e dello statalismo del decisionismo autoritario, quando non dittatoriale". In questa illuminante valutazione si disvela, infatti, il conflitto storico-politico tra stato di diritto e stato costituzionale, esprimendosi la lotta del costituzionalismo contro il paradigma della soggezione acritica, perché avalutativa, al diritto vigente in quanto prodotto della legge figlia di una sovranità illimitata, quindi anche contro il formalismo scientifico avalutativo che, in quanto tale, si esprima in chiave legittimante il regime politico dominante qualunque siano i fini ispiratori della sua azione ${ }^{52}$.

II giurista ufficiale del regime nazista, quale KronJurist, fu infatti accusato di positivismo in quanto artefice di una teoria del diritto funzionale all'affermazione delle categorie culturali a sostegno del nuovo regime, regime come noto ispirato da una idea di costituzione materiale ${ }^{53}$ sintetizzata nel führerprinzip e, paradossalmente, schierato contro ogni teoria formalista che pretendesse di iscrivere il potere arbitrario del sovrano in procedure legali predefinite e quindi in un quadro di limiti giuridici. In questa accezione più generale, quindi, il positivismo descrive quella corrente di pensiero tesa a sostenere di fatto, con le proprie categorie teoriche, il regime politico dominante, schiacciando la propria analisi critica sul diritto positivo vigente, quali che ne siano la forma di manifestazione ed i principi ispiratori posti dal regime ed i valori ${ }^{54}$ a fondamento dell'ordinamento, compreso l'antiformalismo esasperato ${ }^{55}$. Una versione del positivismo, quindi, radicalmente nemica del formalismo, con un'evidente rovesciamento di paradigma. L'antiformalismo diviene strumento indispensabile per poter travolgere l'esistente giuridico al fine di rinnovare i fondamenti del sistema, affrancando giudici, pubblici amministratori ed operatori del diritto in genere da ogni forma di ossequio verso il diritto vigente al momento di avvio del processo rivoluzionario, al fine di "rieducarli" ai nuovi valori ispirati dal regime, ed epurando di necessità - ed ovviamente - le stesse facoltà giuridiche ${ }^{56}$. II nuovo spirito dovrà soppiantare il vecchio diritto e così i giuristi positivisti - nel senso di serventi il nuovo diritto positivo ${ }^{57}$ - dovranno elaborare teorie della costituzione materiale nelle sue varie accezioni, salvo poi professare in un secondo momento un rinnovato ossequio verso il diritto legislativo quando quest'ultimo avrà assunto i nuovi contenuti di valore professati ed arbitrariamente imposti dal nuovo regime politico, divenuto ormai dominus indiscusso degli 
strumenti legali di produzione del diritto e conseguentemente della nuova legalità ${ }^{58}$. Così restaurando il tradizionale nesso tra volontarismo e positivismo, nel deteriore abbraccio già denunciato con riferimento alle correnti giuspositiviste di matrice ottocentesca in apertura di questo scritto $^{59}$.

Di qui, allora, la critica antipositivista professata dal costituzionalismo, inteso quest'ultimo come "teoria giuridica dei limiti del potere politico» ${ }^{60}$ e movimento culturale ispirato dalla necessità di sostenere, pertanto, le ragioni della lotta contro i pericoli del volontarismo, anche laddove quest'ultimo si presenti mascherato da diritto scientificamente oggettivato e, come tale, politicamente neutrale. In questa fase storica il formalismo, infatti, non è affatto libero, neanche teoricamente, dall'autorità del comando di chi pone il diritto. Ed è in questo specifico contesto storico che si affermarono i principi ispiratori della Costituzione di Weimar ${ }^{61}$ e che, con le costituzioni lunghe, rigide e garantite approvate subito dopo la seconda guerra mondiale, lo Stato costituzionale nell'accezione contemporanea si tradusse in diritto positivo ${ }^{62}$.

Del resto era stato proprio Kelsen a rifiutare a priori, in una con la teoria decisionista di Schmitt, l'idea di costruire una teoria del diritto fondandola sul volontarismo e sullo statualismo. Rifiuto implicante il rigetto della teoria imperativistica del diritto a favore di un modello ideale di normativismo in funzione della neutralizzazione e depurazione del diritto da ogni forma di arbitrio naturalmente insita nel modello volontarista di ordinamento, con ciò determinando una svolta storica nelle dottrine del costituzionalismo. E' proprio con Kelsen, infatti, che 'La sovranità diventa necessariamente un predicato delle (sole) norme giuridiche $e$, in particolare, della norma fondamentale, cioè della Costituzione ${ }^{, 63}$. Portato alle estreme conseguenze dal costituzionalismo contemporaneo, il paradigma kelseniano viene così neutralizzato da ogni implicito residuo volontarista per contrastare ogni possibile atteggiamento di lealtà verso il regime politico dominante che quella lettura del positivismo legalista lasciava ancora aperta. Con la positivizzazione del limite costituzionale del potere di porre il diritto, nella disponibilità dell'autorità politica, cade infatti la possibilità di chiudere il discorso del diritto sulle sole scelte arbitrarie del legislatore, ponendo le basi giuridiche di un giudizio di legalità costituzionale, in sintesi di validità, delle stesse norme giuridiche positive di rango sub-costituzionale.

E' in questa forma che, appunto, il costituzionalismo del secondo Dopoguerra si incarica di correggere tutti i limiti della teoria kelseniana del diritto, evidenti solo che si volga lo sguardo alla specificità del diritto costituzionale positivo, sviluppando l'analisi secondo l'oggetto ed il metodo propri di tale disciplina di studio, per orientare la riflessione nel quadro delle sue specificità rispetto alle metodologie proprie, invece, del positivismo analitico e logicistico. Lo studio del diritto costituzionale positivo impone, infatti, di osservare il diritto costituzionale vigente come tale, evitando di assumere in sua vece un determinato modello teorico post-kelseniano come oggetto di riflessione ${ }^{64}$. Tra i possibili corollari della dottrina pura del diritto, seppur al costo di qualche semplificazione, emergevano infatti l'affermazione della democrazia come metodo ${ }^{65}$, mera forma, procedimento qualificandosi il diritto stesso come pura forma. L'indifferenza ai valori ed ai contenuti del diritto, il rifiuto dell'idea che la costituzione condizioni nomostaticamente il contenuto del diritto legislativo ${ }^{66}$, evitando ogni confronto tra i contenuti delle norme lungo lo stufenbau in favore della nomodinamica; l'assunzione che la norma superiore attribuisca una mera competenza, senza però condizionare l'autorità che in base a tale competenza dovrà porre poi la norma di condotta quanto al suo contenuto. II fenomeno giuridico non si assume' perciò, vincolante quanto ai propri contenuti, quanto ai fini ed agli sviluppi dell'ordinamento giuridico. La costituzione non ha pertanto natura prescrittiva quanto ai 
programmi, ai valori, ai fini che pure pretenderebbe di porre agli sviluppi futuri dell'ordinamento giuridico. Da queste premesse si chiarisce in limine la assoluta incompatibilità tra formalismo logicista e costituzionalismo democratico, aperto al pluralismo istituzionale e dei valori ${ }^{67}$.

Come è già da tempo stato rilevato dalla più attenta dottrina costituzionalistica, infatti, il formalismo kelseniano non è affatto politicamente neutrale bensì conforme ad un'ideologia giuridica ben definita e servente il regime politico del liberalismo borghese. E', in sostanza, il diritto del sovrano assoluto, prima, e del parlamentarismo omogeneo ottocentesco, poi. Si predica dello studio del diritto nel suo esclusivo denotato formale rifiutando l'osservazione della norma secondo il suo contenuto e così rinunciando alla critica dell'esistente ${ }^{68}$. Scompaiono dall'indagine dello studioso il piano della giustificazione e della legittimazione dell'ordinamento e delle norme giuridiche; il fenomeno giuridico diviene un mero dato di fatto. Di qui l'espressione forse più forte contenuta nel più volte citato saggio critico di Baldassarre: "Per il positivismo giuridico, la «scienza» del diritto consiste proprio in ciò: nella tautologia, nella ripetizione circolare di ciò che l'Autorità ha «posto» (positum) come suo comando, magari conquistando a questo l'assenso degli altri. In una parola, il giuspositivismo è la variante giuridica dell'autoritarismo politico,69.

In questo senso, allora, il positivismo legalistico diviene storicamente funzione del volontarismo, ed è perciò che il costituzionalismo vi si oppone culturalmente ed ideologicamente $^{70}$. Ad imporlo saranno la pretesa esautorazione del Parlamento, l'annullamento del conflitto sociale, il mascheramento delle ragioni dei singoli individui, dei cittadini, del popolo: a rilevare è soltanto la legge, perché incarna la volontà del sovrano ${ }^{71}$.

'La distribuzione secondo scelte politiche delle fonti tra diversi organi e soggetti caratterizzati diversamente quanto a composizione politica già negava, da sola, la pretesa purezza della teoria kelseniano del diritto. Ma smaschera anche l'altra pretesa di tale teoria, quella di definire la distribuzione delle fonti come sistema oggettivo di legittimazione ${ }^{, 72}$. Le costituzioni contemporanee, all'opposto, non legittimano quale fonte unica ed assolutistica del diritto positivo qualche autorità politica, prescrivendo invece limiti di competenza, forma e procedura nonché vincoli di contenuto financo alla legge del Parlamento democratico-rappesentativo, e costruendo l'ordinamento giuridico come un sistema di poteri diviso, organizzato e ordinato in un quadro di limiti giuridici e reciproci bilanciamenti tra le istituzioni dotate di poteri normativi. Non così, invece, per il positivismo ottocentesco. E' stato Carlo Roehrssen a riflettere sull'origine politica 'dell'isolamento della Staats- und Rechtslehre dal resto delle scienze morali o sociali... Il diritto diventa, se lo si guarda a nudo, privato cioè di tutti i suoi orpelli, una tecnica di potere" In questa prospettiva allora "lo stato e il diritto esistono originariamente...la giuridicità è una categoria trascendentale...il pensare «giuridicamente» è un modulo a-storico, atemporale, che è l'unico modo di pensare la società,"73.

Ma allorché la legalità costituzionale si è positivamente sovrapposta alla legalità legale, il vecchio diritto positivo ha dovuto misurarsi a sua volta con un parametro giuridico non più autoreferenziale, aprendo la storia del positivismo ad una prospettiva di crisi del vecchio modello di formalismo legalistico. Se per la teoria positivista 'fll «regno dei fini»" coincideva totalmente, per la parte pubblica, con la politica e, per la parte privata (...), con la morale individuale ${ }^{, 74}$ ed era quindi escluso dall'osservazione scientifica, con l'avvento delle costituzioni rigide l'ordinamento ha storicamente positivizzato fini e valori politici imponendone l'attuazione al legislatore, la cui discrezionalità ne risulterà perciò limitata in quanto vincolata al loro perseguimento. II diritto positivo diviene quindi oggetto di 
valutazione scientifica rispetto ai fini dell'ordinamento, in quanto normativizzati, ed "ai problemi di legalità si sovrappongono, in funzione validante, quelli della legittimità,"75.

E' quindi necessario professare la definitiva uscita dal formalismo con conseguente approdo ad una qualche teoria realistica? Trovo che questo sia un passaggio estremamente delicato della riflessione. Quale può essere il vincolo formale posto da un diritto costituzionale positivo, sì, ma strutturalmente composto non da sole regole, bensì anche da principi 0 , addirittura, valori ${ }^{76}$

Certamente la Costituzione diviene 'fonte di legittimazione del potere politico e quindi misura suprema della legalità,"77 e la scienza del diritto pubblico dovrà valutare la compatibilità della stessa legalità ${ }^{78}$ con la Costituzione ed $\mathbf{i}$ suoi principi. La struttura normativa della Costituzione, non più composta soltanto da regole ma anche da principi, valori, fini dell'ordinamento, tutti in egual modo giuridicamente prescrittivi ${ }^{79}$, non consente ormai più un approccio di analisi meramente formalistico. L'interpretazione ne diviene funzione essenziale, ma non può ridursi anch'essa ad esercizio analitico, a formalismo, a logicismo. Per questo diviene indispensabile che la scienza del diritto costituzionale si interroghi sui rapporti tra la costituzione quale fonte di legittimazione giuridica (positiva, appunto) dell'ordinamento e diritto positivo di rango legislativo, tra cause di giustificazione del potere legale e sua esplicazione normativa, sua declinazione attraverso il diritto. E' l'oggetto di studio del diritto costituzionale ad imporre questa prospettiva di analisi, il che rende impossibile limitare l'osservazione alla struttura formale del diritto, prescindendo cioè dai suoi contenuti e dalla compatibilità di questi con il nuovo parametro costituzionale, parametro normativo al quale ormai l'ordinamento stesso imputa una valenza positiva, prescrittiva. La storia del diritto positivo non consente più, infatti, di assumere la Costituzione come un dato presupposto, un a priori di fatto, vuoto di contenuti di valore, politicamente neutrale, esterno al diritto ed alla osservazione scientifica ${ }^{80}$.

Se quindi il costituzionalismo contemporaneo avversa l'ipotesi che la scienza giuridica possa assumere la configurazione di un metodo di indagine meramente descrittivo dell'esistente, quale potrebbe scaturire se del caso anche dal formalismo proprio dei modelli di giuspositivismo analitico, le due prospettive di studio non possono che collocarsi in posizioni reciprocamente inconciliabili, professando l'assoluta incomunicabilità dei rispettivi dati di osservazione. Ma questa ipotesi non dovrebbe potersi neanche dare in concreto, laddove si assuma - come qui si assume - che gli oggetti della riflessione delle due discipline, o che i punti di osservazione illuminati dalle due metodiche, siano diversi. Che gli studiosi dell'una e dell'altra categoria dei giuristi positivi, da una parte, e dei filosofi analitici e teorici dell'interpretazione, dall'altra, osservano oggetti differenti. Che per gli uni e per gli altri l'espressione «diritto positivo» qualifica cose diverse. Questa premessa, che rappresenta anche la conclusione del processo di comparazione tra i diversi punti di vista qui confrontati, dovrebbe altresì giustificare l'impossibilità per la scienza costituzionalistica di accettare - per la sua vocazione all'osservazione del concreto, reale giuspolitico, del diritto positivo effettivamente esistente, vigente nel momento dato - le costruzioni teoriche condotte su sistemi ideali, quali quelli che si assume dovrebbero essere composti, per rispondere agli specifici requisiti strutturali richiesti, soltanto da regole compiute; caratterizzati cioè, ove possibile, dall'assenza di principi; in cui addirittura si possa predicare l'esclusione di un ruolo stesso dell'interpretazione, ecc. oppure e - solo all'apparenza - all'opposto, le teoriche dell'interpretazione formaliste che astraggono il discorso giuridico nel logicismo, nel formalismo pratico, nel legal reasoning e nell'analisi del linguaggio e dell'argomentazione giuridica. 


\section{TEORIA GENERALE DEL DIRITTO E POSITIVISMO GIURIDICO}

Che la dottrina classica del positivismo giuridico abbia dovuto affrontare una profonda crisi di metodo a causa dell'avvento delle costituzioni lunghe, rigide e garantite del secondo Dopoguerra è ormai un dato acquisito nella stessa sistematica filosoficoanalitica $^{81}$.

Ma anche il costituzionalismo va assunto, in questa prospettiva, quale teoria del diritto storicamente determinata ${ }^{82}$, che solo negli sviluppi più recenti oltrepassa i limiti di una teoria giuridica della democrazia liberale per distinguersi radicalmente dal positivismo al quale storicamente succede, salva la parentesi di Weimar, nel secondo Dopoguerra ${ }^{83}$.

A riprova di ciò basti richiamare la nota analisi critica sui riflessi prodotti sullo stato di diritto dall'avvento dello stato costituzionale, fondata sulla pretesa incompatibilità tra le dottrine ricostruttive dei due sistemi giuspolitici - tra loro asseritamente inconciliabili - . L'esito della riflessione si sostanziò, nei notissimi saggi di Ernest Forsthoff raccolti nel volume dal titolo Stato di diritto in trasformazione ${ }^{84}$, nella denuncia conseguente di una vera e propria crisi di metodo delle scienze giuspubblicistiche. L'illustre studioso si interrogava infatti sulle conseguenze, per la riflessione scientifica e per la stessa teoria del diritto, della conclamata incompatibilità tra stato di diritto e stato sociale, il primo da intendersi necessariamente in senso formale, a difesa dello status quo economico e sociale, ed il secondo da reinterpretarsi quindi in difesa del primo. La professione rigorosa del metodo giuspositivistico in difesa delle sue implicazioni ideologiche giungeva, in quella riflessione, fino a negare effettività storica al costituzionalismo contemporaneo, rifiutando ogni conseguente prescrittività alle nuove disposizioni costituzionali ${ }^{85}$, al fine di continuare a fondare il sistema giuridico sulla "legge dello stato di diritto...dando giusto peso alla natura legislativa della costituzione" "norme costituzionali ineseguibili'"87. Tale atteggiamento metodico avrebbe, così, potuto garantire la protezione della "natura legislativa della costituzione", legislativa nel senso formalistico dell'espressione, riferita cioè alla struttura delle sue proprie regole. L'attribuzione di senso normativo ai principi ed ai valori costituzionali ${ }^{88}$ avrebbe, altrimenti, portato "alla disintegrazione della legge costituzionale" dissoluzione del diritto costituzionale formale-normativo nella casistica ${ }^{90}$ (...) trova riscontro anche nel modo di funzionare della Corte costituzionale (...) La distruzione delle qualità formali e razionali del diritto legislativo costituzionale equivale anche allo spossessamento della scienza giuridica e del giurista nell'ambito della costituzione ${ }^{, 91}$.

L'avvento dello stato costituzionale ha prodotto, altresì, una vera e propria crisi di prospettiva $^{92}$ nel pensiero dello studioso che aveva addirittura concorso alla fondazione della scuola analitica italiana, Norberto Bobbio. Nei saggi raccolti nel volume Dalla struttura alla funzione. Nuovi studi di teoria del diritto ${ }^{93}$, l'illustre studioso denunciò più volte i limiti, i difetti, le insufficienze del formalismo giuridico a fronte della concreta struttura normativa, della rilevanza dei fini e della ritrovata centralità della funzione del diritto, del ruolo delle disposizioni costituzionali del 1948 nella programmazione di una trasformazione sociale ed economica attraverso il diritto. Il diritto positivo veniva infatti ora, come dire, finalizzato, funzionalizzato ad obiettivi prescritti - programmati - dalla Costituzione, con ciò mettendo in crisi le categorie del formalismo giuridico ${ }^{94}$. 
Va però precisato che di teoria funzionale del diritto Bobbio parla in due differenti accezioni, la prima con riferimento ai fini generali del diritto in sé, come l'ordine e la pace sociale; la seconda invece, più specificamente, dando rilievo proprio ai concreti fini dell'ordinamento come opzioni politiche positivizzate attribuendo, così, alla costituzione un ruolo controfattuale in riferimento al tipo di società da essa progettato. Certo anche nella elaborazione della teoria funzionale, costruita sulla base di un sistema di sanzioni positive e di una funzione promozionale del diritto Bobbio non abbandona la prospettiva analitica ${ }^{95}$. Ma la denuncia di una crisi di effettività della prospettiva meramente normativistica del diritto resta il dato più importante. La necessaria dimensione funzionale della costituzione assume, invece, rilievo centrale nella prospettiva del costituzionalismo, laddove la costituzione "realizza il progetto di costituzionalizzazione dei conflitti", funzione della costituzione, "costruzione integralmente storica e relativa delle vicende umane", laddove orientata alla legittimazione dei conflitti, "impone di prendere in considerazione il suo contenuto,97 abbandonando, come accennato, la lettura dell'ordinamento nella prospettiva meramente formale.

In base a queste premesse è forse, allora, opportuno portare la nostra attenzione sulla nozione stessa di "teoria generale del diritto", espressione questa che non ritengo si possa utilizzare quale paradigma concettuale avulso dalla storia. La "teoria generale" è, infatti, il prodotto culturale di una specifica fase di sviluppo della scienza giuspubblicistica, quella della costruzione dell'ordinamento dello stato unitario, categoria elaborata quindi nel corso dell' $800^{98}$ : 'Nella moderna teoria giuridica dello stato è tradotta tutta una concezione politica; concezione politica che si sottrae appunto alla possibilità di discussione e di verifica, proprio trasformandosi in diritto",99.

La crisi della teoria generale seguita storicamente all'avvento delle costituzioni lunghe, rigide e garantite del secondo Dopoguerra impone quindi una rielaborazione di sistema ${ }^{100}$, nuovo compito per la scienza giuridica che dovrebbe quindi misurarsi con la necessità di elaborare una nuova teoria del diritto, conforme al modello giuridico positivo attualmente vigente ${ }^{101}$. Ma qui la riflessione deve necessariamente interrompersi, per limitarsi a segnalare le difficoltà oggettive della ricostruzione di un nuovo paradigma teorico, di una nuova teoria del diritto dello Stato costituzionale, compatibile con la nuova struttura del diritto positivo, composto non di sole regole ma da differenti canoni, da valori e principi che dovranno però essere comunque oggetto di attenzione scientifica da parte della contemporanea declinazione delle dottrine del positivismo giuridico in quanto positivizzati, come dire?, normativizzati. L'approccio qui seguito, lo si è segnalato fin dalle premesse, sconta molto il dato normativo concreto, il modo cioè in cui il diritto effettivamente è oggi posto, scritto, normativamente declinato, per tacere degli effetti che sulla teoria del diritto dovrebbero derivare dalla crisi non solo del regime di stato democratico ma della stessa forma Stato quale sintesi dell'ordinamento giuridico.

Come utilizzare, ad esempio, la "cassetta degli attrezzi" del giuspositivista allorché il diritto positivo assuma le forme proprie della disciplina europea dei diritti fondamentali? Come gestire un modello normativo asseritamente di rango costituzionale ma composto da clausole generali appositamente strutturato - quale quello europeo - per lo sviluppo successivo di un diritto ad elaborazione giurisprudenziale? E come trattare metateoricamente lo sviluppo asistematico di un ordinamento costruito secondo le categorie del "disordine"? ${ }^{102}$ Per tacere degli attuali sviluppi dell'ordinamento giuridico italiano ormai vittima di un protagonismo delle fonti governative adottate ultra vires ${ }^{103}$ nel deliberato intento di interferire con specifiche vicende giudiziarie in corso e come tali idonee a travolgere lo stesso principio della separazione dei poteri. Quale teoria del diritto 
potrebbe infatti sistemare la pretesa del governo di interferire con propri atti normativi generali ${ }^{104}$ su decisioni relative a singoli casi già in pendenza di giudizio davanti ad un giudice comune? Quale nomodinamica potrebbe, infatti, sostenere questo confuso intreccio di ruoli?

In realtà si potrebbe procedere ancora oltre nel denunciare le difficoltà di definizione di una teoria del diritto conforme al modello di diritto positivo vigente, spingendosi verso un'ulteriore analisi critica, orientata lungo il paradigma dei rapporti tra giuspositivismo e linguaggio giuridico (inteso come linguaggio di chi materialmente pone il diritto). Altro essendo l'esito dell'applicazione del metodo formalista per la comprensione e la interpretazione di un diritto scritto con un linguaggio giuridico omogeneo e condiviso in quanto frutto della costruzione dell'ordinamento ad opera di una classe, politicamente e culturalmente omogenea di giuristi (e di legislatori!); altro, invece, misurarsi con il linguaggio giuridico contemporaneo, estremamente più complesso in quanto prodotto di negoziati, di sintesi di diverse culture giuridiche, spesso di opposti paradigmi politici e della propaganda, non raramente imponendo di ricostruire in via interpretativa concetti ed istituti che il legislatore non ha potuto far altro che declinare normativamente mediante l'utilizzo di perifrasi. Norme giuridiche scritte in tale modo rendono non più funzionale, o comunque poco utile, il ricorso alle categorie interpretative del formalismo. E' il problema di utilizzare normativamente un linguaggio strutturalmente non normativo.

Come di recente osservato, allora, l'attenzione della teoria del diritto deve muovere più che dalla struttura dell'ordinamento, proprio a causa delle funzioni che il sistema istituzionale deve affidare al diritto al fine di rendere determinato tutto quanto sia di incerta configurazione per causa di "antinomie, indeterminazione, incommensurabilità"105 in quanto non preventivamente risolto nella composizione materiale delle norme giuridiche. Se il diritto, sul modello nomodinamico kelseniano, è 'q'istituzione di poteri normativi: poteri, istituiti e disciplinati da norme, di produzione o applicazione di norme" procedure regolate da norme, anche la soluzione dei casi difficili deve trovare nel diritto stesso le proprie forme ed i propri limiti, a prescindere dal riferimento a principi e valori materiali. Per quanto la struttura normativa dell'ordinamento positivo sia aperta a causa del processo di legittimazione dei conflitti ${ }^{107}$, sostenuto dalle costituzioni democratiche contemporanee, il diritto deve fornire i criteri di composizione e bilanciamento necessari decidendone l'imputazione, le procedure, le forme, ed i limiti di contenuto della discrezionalità attribuita a chi sia depositario di tale potere: sia esso il legislatore, nei limiti di contenuto previsti dalle norme costituzionali o il giudice, come nel sistema comunitario di garanzia dei diritti fondamentali.

Come spero di riuscire a chiarire nell'ultimo paragrafo, è allora necessario concorrere alla costruzione di una possibile teoria normativa della costituzione quale elemento fondativo dell'ordinamento, di cui la costituzione ha programmato almeno in parte lo sviluppo normativo mediante la prescrizione di fini e valori. La costituzione vigente deve essere, pertanto, assunta come vincolo positivo per i successivi sviluppi legislativi, istanza di canalizzazione e neutralizzazione del mutamento che sia funzionale alla recezione delle istanze politiche e sociali. 'La costituzione nata e perfezionata come rigida proprio per soddisfare il bisogno di stabilità e certezza dei consociati, tenta di trovare così il suo equilibrio nella procedimentalizzazione del mutamento, nella istituzionalizzazione della complessità e della variabilità, nella riaffermazione del carattere della certezza del diritto (...) non più nei contenuti, ma, principalmente, nella forma dei mutamenti, ${ }^{\text {,108 }}$. Come di recente osservato da Luciani, infatti, "nella Costituzione le regole del gioco sono fissate 
«allora per ora» (...) il testo esprime l'accordo fondativo di strutturazione dell'ordinamento"109.

In questa chiave è, allora, possibile richiamare la proposta di conclusione formulata in precedenza. Nella prospettiva di metodo prescelta quale linea di sviluppo del costituzionalismo contemporaneo a dominare è, infatti, la sintesi tra forma e contenuti di valore, sintesi declinata secondo il paradigma appena descritto. Pluralismo valoriale e realismo, ma costretto quest'ultimo nelle forme e nelle procedure prescritte, in quanto positivizzate, normativizzate. Forme e procedure però di cui il sistema normativo prescrive il rispetto sostanziale e non meramente formale. L'utilizzo strumentale di tali elementi normativi, l'arbitraria forzatura delle une e delle altre al fine di ottenere un risultato formalmente compatibile con la Costituzione, ma sostanzialmente in frode ad essa finirebbe infatti con il violare e contraddire comunque ambedue gli elementi normativi, prescrittivi quanto alla loro intima sostanza e non solo quanto alla veste formale dei mutamenti per come da essi definita. L'ossequio per le forme dei mutamenti, cioè, non potrebbe giustificare ad esempio il superamento del regime democratico verso un nuovo sistema di impianto monocratico, laddove il rispetto del procedimento di revisione costituzionale non sarebbe affatto garantito dall'approvazione da parte della sola maggioranza di governo della relativa legge di riforma, a pena di compromettere il significato più profondo del carattere normativo della Costituzione.

Certo una volta formulata questa professione di metodo, individuata cioè la imprescindibile necessità di declinare una teoria del diritto costituzionale positivo idonea a comprendere la fenomenologia giuridica del secondo Novecento con costante attenzione al reale giuspolitico, anche al fine di garantire al fondamento del sistema normativo una giustificazione in termini di effettività dell'ordinamento - e della Costituzione - resta pur sempre aperto il problema del valore e del ruolo del metodo giuspositivistico nel rinnovato contesto teorico.

L'apertura della dottrina e della giurisprudenza alla teoria dei valori ed al bilanciamento quali tecniche interpretative, decisorie ed argomentative lascia infatti troppo spazio ai pericoli dell'interpretativismo, dell'arbitrio degli interpreti nell'attività di ius dicere e, quindi, di ius ponere. La consapevolezza delle nuove frontiere problematiche non può infatti giustificare l'abbandono del paradigma normativo tout court, che spesso le dottrine più antiformaliste sembrano professare. Penso, naturalmente, all'impianto teorico professato dalle dottrine di studiosi quali Zagrebelsky e Silvestri nei saggi più sopra citati, pur nell'evidente necessità di segnalarne le importanti differenze di impostazione, ed in misura diversa anche Baldassarre e Modugno, fino a profetizzare il trapasso da una democrazia costituzionale politica, in quanto pluralista, ad una forma di Stato che, per semplificare, intitolerei alla "democrazia dei custodi',"10.

\section{PER UN DIRITTO COSTITUZIONALE NORMATIVO}

II punto più debole di quella che, per semplicità, qui chiamo 'teoria dei valori" resta tuttora la difficoltà di salvaguardare, in essa, i "valori" appunto della certezza del diritto della prevedibilità, cioè, della decisione del giudice - e del diritto positivo, del dato normativo testuale. Diversi anni fa, in un breve saggio ${ }^{111}$, avevo già provato a prendere posizione sul problema, alla difficile ricerca di una possibile conciliazione tra il necessario contributo pratico ${ }^{112}$ dell'ermeneutica ed il costituzionalismo normativo, riflessione alla quale qui mi limito a fare rinvio. A quelle considerazioni aggiungo ora soltanto qualche 
ulteriore elemento, in relazione ai caratteri del costituzionalismo e del valore della certezza del diritto nei sistemi di Common Law, che spesso le teoriche (e le pratiche) aperte all'affermazione di un diritto casistico di matrice giurisprudenziale assumono quale ambiente culturale di riferimento.

Creare diritto attraverso la pratica del bilanciamento tra valori potrebbe, infatti, contenersi entro una soglia che eviti il trionfo dell'interpretativismo più spinto - e quindi dell'arbitrio - laddove almeno si codificasse nei fatti un rigoroso vincolo del precedente ${ }^{113}$ a far da paradigma argomentativo al fine di giustificare, appunto, ogni decisione...secondo diritto obiettivo. Nell'accurata riflessione sui dati della ricerca storica e sul pensiero di Albert Venn Dicey, in relazione ai rapporti tra la Rule of Law, la sovranità del Parlamento ed il ruolo della tradizione nel Common Law anglosassone, Pietro Costa ${ }^{114}$ segnalava proprio questo profilo, soffermandosi sul Common Law quale strumento di conservazione, paradigma di resistenza dell'ordinamento contro il mutamento arbitrario dei suoi contenuti.

Dicey aveva, infatti, subito rilevanti critiche dalla dottrina a lui contemporanea con l'accusa di aver aperto le porte, nel suo saggio fondamentale ${ }^{115}$, al volontarismo positivista sostenuto dalla professata sovranità del Parlamento (The Law of the Constitution), la cui autorità politica avrebbe costituito il fondamento del diritto anglosassone contro la Rule of Law protetta dal Common Law. Scrive Costa che 'il trattato di Dicey fu concepito, scritto, rivisto, letto e discusso in un ambiente, quello dei giuristi inglesi, tra diciannovesimo e ventesimo secolo, intriso di austinianismo", nel senso di "positivismo giuridico di Austin"116. Ma se si procede distinguendo la ricostruzione storica e la dottrina di Dicey dall'impostazione legicentrica à la Austin per ricondurle alla tradizione di Common Law, ci si avvede come in realtà la sovranità del Parlamento, secondo la ricostruzione dell'illustre studioso, non potesse mai svolgersi se non in conformità con lo spirito della costituzione (la constitutional morality ${ }^{117}$ ), per come interpretato e garantito dalle Corti inglesi. I canoni della Rule of Law a protezione dei diritti costituzionali, infatti, imponevano alle Corti di non dare esecuzione alle leggi difformi dal Common Law, ma di interpretarle in modo tale da "amalgamare la volontà del Parlamento con la tradizione costituzionale....neutralizzando il volontarismo insito nel principio della sovranità del Parlamento". "Hale sostiene infatti che solo il Parlamento ha il potere di produrre nuovo diritto, ma questo prodotto ha un impatto e un significato limitati se non è «incorporato» nel Common Law (...). La teoria classica del Common Law si fonda sull'idea che "attraverso l'interpretazione» i giudici esercitano un «controllo» costante sulla legislazione". In tal modo il volontarismo positivistico veniva ricondotto nei canoni della legalità garantita dalla costituzione inglese e protetta dalle Corti, inibendo alle maggioranze parlamentari occasionali di adottare leggi in conflitto, appunto, con the Common Law of the Land a protezione dei diritti fondamentali dei cittadini, e rendendo il diritto costituzionale "quasi impermeabile agli eccessi del volontarismo legislativo,"118.

Secondo le categorie della tradizione costituzionale inglese, quindi, i canoni del formalismo vengono declinati, come dire?, all'inverso rispetto all'esperienza giuridica continentale, laddove consentano ad una legge formalmente valida di essere giudicata secondo i suoi contenuti al cospetto del Common Law al fine di valutarne la conformità ad un diritto costituzionale di matrice giurisprudenziale. Diritto giurisprudenziale in cui si sostanziano, però, i valori della stabilità e della certezza dei remedies a protezione dei diritti dei singoli individui anche contro gli eccessi arbitrari del legislatore. Stabilità e certezza garantite, appunto, da un rigoroso rispetto del vincolo del precedente, matrice giuridica del legalismo inglese, legalismo però di matrice giudiziaria. Constatazione, questa, che imporrebbe un'attenta analisi critica sui rapporti tra diritto e morale in quel 
contesto culturale, per attribuire alla nozione di moral un significato che, nella giurisprudenza come scienza pratica trova forse il suo significato più profondo proprio nei contenuti normativi del Common Law of the Land. Analisi che, però, esula dalla presente riflessione.

In conclusione vorrei, infine, riprendere alcune questioni derivanti dagli elementi caratterizzanti quella corrente di pensiero che, credo in termini meramente descrittivi ${ }^{119}$, viene oggi definita con il termine di neocostituzionalismo.

In un recente saggio dal titolo Neocostituzionalismo e positivismo giuridico ${ }^{120}$, utile proprio in relazione alla difficile ricostruzione dei complessi momenti di comunicazione tra dottrine costituzionalistiche e teoriche dei filosofi analitici ${ }^{121}$, Susanna Pozzolo giunge, nelle sue conclusioni, ad alcuni momenti di sintesi che potremmo riassumere in questo modo: il neocostituzionalismo si connette alla centralità della teoria dei valori e del bilanciamento quali elementi caratterizzanti la riflessione teorica di alcuni costituzionalisti e teorici del diritto, con conseguente perdita di effettività dei canoni del formalismo giuridico e, allo stesso tempo, aumento dei rischi connessi di arbitrio degli interpreti, divenuti sacerdoti del diritto; la costituzione, i suoi fini ed i valori in essa professati assumono carattere pervasivo nel sistema giuridico complessivamente inteso. Nella riflessione della Pozzolo la costituzione viene, quindi, ancora considerata un a-priori rispetto al diritto positivo, starei per dire di matrice giusnaturalistica ${ }^{122}$, che condiziona moralmente (politicamente) l'ordinamento ma senza farne ancora parte, senza potersi individuare come suo elemento costitutivo, diritto positivo essa stessa; da ciò l'insistenza ad evitare che la riflessione dei giuristi ridondi nella valutazione delle norme giuridiche formalmente valide in ragione del loro contenuto.

Alcune tra le critiche così formulate all'indirizzo della riflessione costituzionalistica contemporanea sono, in effetti, condivise anche da parte importante della stessa dottrina costituzionalistica, specialmente in relazione ai rischi di soggettivismo ${ }^{123}$ connessi con la pretesa centralità della teorica dell'interpretazione per valori. L'affievolimento del tenore di vincoli testuali ${ }^{124}$ professato, almeno in parte, da tale dottrina rischia infatti di "pregiudicare quella neutralizzazione dei conflitti politici (...) la quale costituisce uno dei principali obiettivi del moderno costituzionalismo,"125.

Ora la sintesi ideologica, di metodo e di teoria costituzionale - per dirla con Bobbio che a questo punto riterrei di poter qui formulare, mi porta a richiamare quali suoi elementi essenziali gli assunti professati nel programma di politica culturale sostenuto dalla Rivista Costituzionalismo.it ${ }^{126}$. Con una forte attenzione al positivismo giuridico nell'accezione contemporanea, per l'indispensabile riferimento al testo della Costituzione, al carattere essenzialmente normativo, prescrittivo della Costituzione, ma nella consapevolezza che la Costituzione medesima si presenta quale opzione politica storicamente determinata ed inverata nella storia, che dalle rivoluzioni settecentesche in poi ha significato - e tuttora significa - ruolo contro-fattuale del diritto costituzionale in quanto strumento giuridico di limitazione del potere ${ }^{127}$ e garanzia giuridica dei diritti individuali. La teoria costituzionale professata esprime, pertanto, un punto di vista ${ }^{128}$.

II diritto costituzionale, infatti, è cosa molto diversa dal diritto pubblico di uno Stato, in coerenza con un principio giuspolitico risalente all'art. 16 della Dichiarazione dei diritti dell'uomo e del cittadino del 1789. Il diritto costituzionale impone, infatti, la valutazione della coerenza degli sviluppi dell'ordinamento ai fini ed ai principi normativizzati nella costituzione medesima, come ricordato fin dall'apertura del presente saggio. 
'Mentre il contrario della validità è l'invalidità, il contrario della normatività è la descrittività; e sono descrittive le costituzioni indifferenti al mantenimento del loro contenuto,129. Di qui il riconoscimento nel metodo del positivismo giuridico assunto nella sua declinazione storica contemporanea, che oggi impone, secondo la costituzione vigente, di accertare e valutare i dati normativi nei significati che assumono nella configurazione materiale della condizione umana, secondo le domande della democrazia $^{130}$. Il positivismo giuridico diviene strumento di lotta per il diritto, contro la legittimazione indiscriminata di qualsivoglia normativa per il semplice fatto di essere posta da un qualche potere, con il conseguente rifiuto dell'assunzione acritica di qualunque contenuto normativo, e il rifiuto altresì di un metodo risolto nella mera esegesi del dato normativo che deve essere, all'opposto, valutato secondo le prescrizioni costituzionali vigenti.

Il diritto costituzionale positivo è oggi, infatti, uno strumento contro-fattuale, frutto delle conquiste di civiltà che la lotta per il diritto ha proposto e propone al fine della trasformazione del reale. Un diritto normativo, prescrittivo, ma non risolto nel formalismo, giusta la sua pretesa di misurarsi con il reale ${ }^{131}$. Positivismo e realismo vanno quindi declinati insieme nel confronto con lo sviluppo storico dei relativi oggetti di riflessione. I valori politici del costituzionalismo, affermatisi nella storia moderna e contemporanea, sono stati infatti normativizzati e costituiscono oggi il paradigma valutativo del diritto positivo a seguire. '"l compito delle costituzioni è quello di chiudere i discorsi intorno alla validità degli atti che pongono il diritto"; intorno "alla legittimità dei comportamenti degli organi costituzionali, ${ }^{\prime 132}$ ed alla legittimità dei contenuti degli atti normativi secondo i propri valori, fini e programmi declinati normativamente ${ }^{133}$. Non potendo prescindere dalla validità delle costituzioni, dalla loro effettività in virtù del riconoscimento loro tributato dalle forze dominanti, dai destinatari dei comandi giuridici, non è ammissibile inoltre "considerare obsoleto il problema del fondamento della validità della costituzione in termini politici complessivi"134.

E' proprio il carattere normativo della Costituzione a porre, quindi, limiti giuridici agli eccessi dell'interpretativismo. Ed è il costituzionalismo prescrittivo, infine, a rifiutare il mero descrittivismo a-valutativo in quanto non conforme al proprio paradigma positivo, dichiarando ad esso non conformi quindi le nuove "scuole dell'esegesi della giurisprudenza". Come esemplarmente insegna Alessandro Pace "ben si può, con riferimento alla nostra Costituzione, utilizzare "criticamente», e cioè con sensibilità al contesto storico e culturale, il "metodo» giuspositivista, il quale, se viene applicato nel rispetto di tali premesse, non designa altro che «il metodo scientifico» ${ }^{135}$.

Sono queste ultime espressioni di un nuovo formalismo? L'ideologia costituzionale implicita nelle teorie del costituzionalismo prescrittivo qui richiamate incarnano oggi all'opposto una nuova forma di giusnaturalismo ${ }^{136}$ ? Della relatività delle qualificazioni e dei giudizi reciproci tra positivisti e giusnaturalisti pur in presenza della consapevolezza del proprio punto di vista si è già detto in apertura di questo saggio. Allo stesso modo si deve dire, credo, della relazione comunicativa tra costituzionalismo e positivismo. Quanto al positivismo analitico, come accennato, il confronto è reso, invece, oltremodo problematico piuttosto dalla diversità dell'oggetto di studio e di riflessione, come credo sia reso evidente proprio dalle considerazioni qui da ultimo formulate. Resta allora, in estrema sintesi, proprio la difficoltà di qualificare in termini assoluti ed oggettivi il proprio punto di vista. Ma forse è proprio questa, a ben vedere, la questione più grande nello studio del diritto costituzionale contemporaneo, la questione che rende indispensabile, per poter guardare Iontano, salire e rimanere sulle spalle dei giganti. 


\title{
POSITIVISMO LEGAL E O ESTUDO DO DIREITO CONSTITUCIONAL
}

\section{RESUMO}

Este artigo tem por objetivo estudar as relações entre Direito Constitucional e Positivismo. Trata-se de revisitar os pressupostos fundacionais e teóricos do Positivismo e o seu ideal de constitucionalismo - traçando também as relações com a teoria geral do Direito para a formação de um Direito Constitucional Prescritivo.

Palavras-chave: Direito Constitucional. Direito Constitucional Prescritivo. Positivismo. Teoria Geral do Direito.

\section{LEGAL POSITIVISM AND CONSTITUTIONAL STUDIES}

\begin{abstract}
This article aims the study of the relationship between positivism and Constitutional Law. The objective is to restudy the positivism theoretical and foundational groundings and it's constitutionalism ideal - marking also the relationships between the general theory of law for the formation of a new prescriptive Constitutional Law.
\end{abstract}

Keywords: Constitutional Law. General Law Theory. Positivism. Prescriptive Constitutional Law.

\section{NOTAS}

* Questo saggio è destinato agli Studi in onore di Alessandro Pace.

Professore ordinario di Diritto costituzionale presso I'Università degli studi di Chieti e Pescara

1 A partire dal noto saggio dall'emblematico titolo Problematica delle libertà costituzionali. Parte generale, ora giunto alla terza edizione, Padova, 2003, spec. 36 ss.; ma a mero titolo anche solo ricognitivo si vedano inoltre, almeno, Le sfide del costituzionalismo ne/ XXI secolo, ora in Id., I limiti del potere, Napoli, 2008, 1 ss.; Metodi interpretativi e costituzionalismo, in Quad.cost., 2001, 35 ss.; Interpretazione costituzionale e interpretazione per valori, in http://www.costituzionalismo.it/docs/interpretazionevalori.pdf , 2/2006, 11 luglio 2006. Ma la riflessione più meditata e completa è forse esposta nelle Considerazioni preliminari al Commento all'Art. 21. La libertà di manifestazione del proprio pensiero, in A. Pace, M. Manetti, Art. 21, in Commentario della Costituzione, fondato da G. Branca e continuato da A. Pizzorusso, Bologna-Roma, 2006, 1 ss.

2 Esemplare dei rischi connessi alle incomprensioni dovute all'assenza di preliminari convenzioni stipulative sull'approccio di metodo seguito, e sui conseguenti necessari distinguo dovuti ai differenti punti di vista, mi sembra il recente dibattito raccolto intorno alle polemiche riflessioni dedicate al tema del positivismo da A. Baldassarre, Miseria del positivismo giuridico, in Studi in onore di Gianni Ferrara, I, Torino, 2005, 201 ss.; Id., Una risposta a Guastini, in Giur. cost., 2007, 3251 ss.; e R. Guastini, Sostiene Baldassarre, in Giur. cost., 2007, 1373 ss. Saggi che si segnalano per il fondamentale contributo critico al tema oggetto della presente riflessione rivelatosi proprio per via del vivace contrappunto tre le opposte impostazioni di metodo.

3 Si pensi alla onnipresente distinzione, declinata come questione dei punti di vista da molti tra i filosofi giuspositivisti, tra discorsi descrittivi e discorsi prescrittivi o normativi in relazione al proprio impegno culturale, a seconda che si "guardi alle regole soltanto dall'esterno" o che, all'opposto, si guardi al diritto dall'interno - "accettando ed usando le regole come guida per la propria condotta", come ci ricorda, di 
recente, S. Civitarese Matteucci, Miseria del positivismo giuridico? Giuspositivismo e scienza del diritto pubblico, in Dir.pubbl., 2006, 685 ss., 686 s., in un saggio estremamente informato che si segnala altresì per l'esemplare coerenza di metodo. Su tale base andrei, forse, anche oltre distinguendo allora il punto di vista dei filosofi da quello dei giuristi, essendo il punto di vista di questi ultimi, secondo tali premesse, necessariamente "interno", M. La Torre, On Two Distinct and Opposing Versions of Natural Law: "Exclusive» versus "Inclusive», in Ratio Juris, 2006, 214. Sulla relativa obsolescenza di tale distinzione mi pare si esprima però, oggi, M. La Torre, Filosofia e scienza giuridica in Norberto Bobbio, in corso di pubblicazione in Ragion pratica, 2010. Si veda peraltro quanto ossenvato da G. Zagrebelsky, Intorno alla legge. I/ diritto come dimensione del vivere comune, Torino, 2009, 85, spec. nt 1) circa l'uso strumentale della preventiva professione di metodo da parte di molti "gius-positivisti".

4 Limitandoci a richiamare gli studi, come dire, di metateoria del metodo giuridico rinvio, per il momento, alla riflessione di Norberto Bobbio nel notissimo saggio su II positivismo giuridico. Lezioni di Filosofia del diritto raccolte da N. Morra, Torino, 1996. Ma si vedano inoltre, almeno, le voci Positivismo giuridico di M.A. Cattaneo in Noviss.Dig., XIII, Torino, 1957, 315 ss. e di F. Modugno in Enc.Dir., XXXIV, Milano, 1985, 448 ss.

5 Non posso che usare questa espressione con molta cautela e senso di approssimazione. Mi conforta, però, l'ampia prospettiva professata da M. Barberis, Breve storia della filosofia del diritto, Bologna, 2004, fin dalla Prefazione del volume. Ma si v., poi, in particolare i capitoli II e VI.

6 Approccio che, come ci ricorda S. Civitarese Matteucci, Miseria del positivismo giuridico?, cit., 687 ss., è divenuto "questione...centrale soprattutto a seguito della spiegazione che ne ha fornito H.L.A. Hart", II concetto di diritto, nuova ed. del 1965, tr.it. a cura di M.A. Cattaneo, Torino, 2002. Ma si veda quanto precisato alla precedente nt. 3).

7 Seguano o meno il c.d. indirizzo analitico. Già questa classificazione è meramente stipulativa e spero lasci intuire, pur in assenza di una possibile linea di demarcazione tassativa, a quali differenze di metodo tra gruppi di studiosi intendo ora fare riferimento. La distinzione mi sembra peraltro già segnalata nel citato saggio di A. Baldassarre, Miseria del positivismo giuridico, cit., 202 ss., almeno con riferimento al positivismo filosofico.

8 Così, infatti, Baldassarre, Una risposta, cit., 3259 'L'essere giuspositivisti riguarda il modo in cui gli uomini intendono e comprendono il diritto, non il modo in cui il diritto viene storicamente posto. Riguarda il diritto come modo di "conoscenza»o di «sapere», non il diritto come fenomeno "oggetto» di conoscenza o di sapere".

9 Nel senso indicato nel testo mi sembrano esemplari gli insegnamenti di L. Ferrajoli, Principia iuris. Teoria del diritto e della democrazia. 1. Teoria del diritto, Roma-Bari, 2007, spec. 21 ss., 41 ss. II sistema costruito dallillustre autore, pur orientato dal suo proprio punto di vista si svolge, infatti, lungo un processo di permanente comunicazione tra i diversi altri punti di vista, acquisendo magistrale importanza proprio in virtù di questa continua giustapposizione. II metodo così (forse semplicisticamente) sintetizzato ispira l'impianto dell'intera riflessione di Ferrajoli ed è altresì consapevolmente descritto dall'autore, tra le altre sedi, alle pagine indicate.

10 Di cui Pace stesso, II metodo di Carlo Esposito, in M. Ruotolo (a cura di), Gli scritti camerti di Carlo Esposito. 1928-1935, Napoli, 2008, 99 ricorda che "non è una singola teoria della natura del diritto" ma citando alla lettera S. Civitarese Matteucci, op.cit, 703 - "un'intera tradizione di pensiero, che copre più di due secoli e comprende un gran numero di teorie spesso tra di loro configgenti anche su questioni centrali".

11 Come è noto il problema è da sempre presente nelle riflessioni sul positivismo, come ci ricorda $\mathrm{N}$. Bobbio, Il positivismo, cit., 234 ss.

12 Ma più in generale mi riferisco alle risultanze concettuali del c.d. 'Legal Positivism". In tesi generale preciso inoltre che non intendo, naturalmente, gli uni e gli altri ascrivibili a due differenti mondi separati ma, anzi, riconosco che gli studiosi concorrono alla riflessione su oggetto e metodo di studio dei giuristi spesso confrontandosi, confondendosi o opponendosi negli approcci anche trasversalmente.

13 II concetto di diritto, cit., 3 ss.

14 Ce lo ricorda M. Luciani, La problematica laicità italiana, in Dem.dir., 2008, 107.

15 Per una analisi critica sulle differenti possibili accezioni del termine rinvio, come è naturale, alla riflessione di U. Scarpelli, Cos'è il positivismo giuridico?(1965), Napoli, 1997,85 ss., 103 ss.

16 U. Scarpelli, op.ult.cit., 165 per il riferimento esplicito al dato del diritto posto, alla sua posizione, al porre, per la differenza di approccio tra "positivismo scientifico" e c.d. "positivismo politico" e per l'opzione dedicata a quest'ultimo approccio. Per quanto si verrà sostenendo più avanti nel corso di questo scritto, si veda, altresì, quanto sostenuto dall'illustre autore in relazione alla necessità di una leale collaborazione del giuspositivista nei confronti della volontà politica espressa dalle nome, appunto, di diritto positivo, 151. Si veda, infatti, quanto sostenuto da M. La Torre, 'Finché la società sarà questa". Neocostituzionalismo e positivismo giuridico: Gustavo Zagrebelsky e Luigi Ferrajoli, in corso di pubblicazione in Materiali per una storia della cultura giuridica, 2010, 1 del dattiloscritto. 
E ben sapendo che anche la categoria dei costituzionalisti è un paradigma nominale che comprende molti e assai differenziati approcci e punti di vista.

18 II concetto di "politico»: testo del 1932 con una premessa e tre corollari, in Id., Le categorie del "politico», tr.it. a cura di G. Miglio e P. Schiera, Bologna, 1972, 89 ss.

19 Nel senso in cui la categoria è utilizzata ad esempio da M. Dogliani, Introduzione al diritto costituzionale, Bologna, 1994, 11 ss.

20 Che con approccio stipulativo qualifico anche come "classico" o "formalista" a seconda che si assuma il punto di riferimento storico o dell'ideologia giuspolitica. Così, ad esempio, G.U. Rescigno, Interpretazione costituzionale e positivismo giuridico, in Dir.pubbl., 2005, 19 ss., spec. 37 ss. con il termine "positivismo giuridico" si riferisce espressamente al paradigma culturale ottocentesco. Si veda, inoltre, F. Spantigati, L'acqua è bagnata e il calore riscalda: il trionfo dell'ovvio, in http://www.grupposanmartino.it/federico/pdf, pti 31 ss. del dattiloscritto Spero di non semplificare troppo ma questo è lo stesso approccio critico che credo di rinvenire nel citato saggio di A. Baldassarre, Miseria, cit., che almeno su questo punto a me sembra sia stato frainteso tanto da S. Civitarese Matteucci, Miseria del positivismo giuridico?, cit. che da R. Guastini, Sostiene Baldassarre, cit. Devo peraltro riconoscere che nella sua Risposta a Guastini, in Giur. cost., 2007, cit., pur impostata sul medesimo approccio storicistico (v. 3255 ss.) del precedente saggio è poi lo stesso Baldassarre ad inseguire Guastini, come dire? nell'approccio metodico di quest'ultimo per articolare la propria riflessione in risposta sul piano della reciprocità polemica.

21 L'argomento è da sempre presente nella riflessione costituzionalistica italiana dell'età repubblicana, basti il rinvio a P. Barile, La Costituzione come norma giuridica, Firenze, 1951 e V. Crisafulli, La Costituzione e le sue disposizioni di principio, Milano, 1952.

22 Lascio qui impregiudicata la questione del se $\mathbf{i}$ «valori» siano riducibili a norme giuridiche e se si dia differenza tra le due tipologie di norme, limitandomi a rinviare per ogni più complessa implicazione alle opposte impostazioni di F. Modugno, ora in Scritti sull'interpretazione costituzionale, Napoli, 16 ss., in particolare nel saggio Interpretazione per valori e interpretazione costituzionale, ivi, 27 ss. e di A. Pace, Interpretazione costituzionale e interpretazione per valori, in www.costituzionalismo.it, n. 2/2006, 11 luglio 2006, Relazioni tenute il 12 maggio 2005 nell'ambito dell'incontro organizzato da G. Azzariti per il Corso di dottorato di ricerca in Diritto costituzionale e diritto pubblico generale nella Facoltà di Giurisprudenza dell'Università "La Sapienza» di Roma. La questione della 'interpretazione per valori" è come noto divenuta oggetto di riflessione sistematica nella dottrina costituzionalistica italiana a seguito dei saggi di L. Mengoni, Diritto e valori, Bologna, 1985; A. Baldassarre, Costituzione e teoria dei valori, in Pol.dir., 1991, 639 ss. (ma si veda ora anche Id., Interpretazione e argomentazione nel diritto costituzionale, in www.costituzionalismo.it, 2/2007, 30/5/2007) e di G. Zagrebelsky, I/ diritto mite. Legge Diritti Giustizia, Torino, 1992 (ed ora Id., La legge e la sua giustizia, Bologna, 2008, spec. $161 \mathrm{ss}$.). Con riferimento esplicito ai diritti costituzionali si ricorda il saggio di R. Bin, Diritti e argomenti. II bilanciamento degli interessi nella giurisprudenza costituzionale, Milano, 1992. Una prima bibliografia essenziale si trova già nel mio Emergenza, interpretazione per valori e certezza del diritto, in Giur.cost., 1993, 3007 ss., spec. 3015 nt. 16. Non posso, inoltre, non richiamare per originalità ed importanza paradigmatica dell'approccio il noto saggio di R. Alexy, Teorie der Grundrechte, Frankfurt am Main, 1994. Si veda ora anche il saggio di A. Longo, I valori costituzionali come categoria dogmatica. Problemi e ipotesi, Napoli, 2007.

23 Sulla relazione tra "positivismo classico" e atteggiamento valutativo rinvio di nuovo alla riflessione di A. Longo, op.ult.cit., spec. 177 ss., anche se non vorrei addentrarmi fin nel cuore delle specificità problematiche delle teorie dell'interpretazione giuridica - e costituzionale in particolare - per la cui complessità sia sufficiente il rinvio alla trattazione sistematica condotta da F. Modugno, Interpretazione giuridica, Padova, 2009.

24 Sempre sistema, come osserva Ferrara con riferimento a ' $q$ tre tipi di pensiero giuridico" di Schmitt (regola, decisione, ordinamento) ed alla connessione tra decisione e regola: 'E' concepibile oggi una norma giuridica senza ordinamento?"' Ma si veda infra.

25 Sul punto è d'obbligo il rinvio alla riflessione di G. Ferrara, Costituzione e revisione costituzionale nell'età della mondializzazione, in Scritti Guarino, II, Padova 1998, spec. 233 ss., 243 ss.; Id., La Costituzione. Dal pensiero politico alla norma giuridica, Roma, 2006, 242 ss.

26 G. Ferrara, Diritto soggettivo, diritto oggettivo. Uno sguardo sugli apici del giuridico, in www.costituzionalismo.it, 3/2008, 14/09/2008, 17. Ho in più occasioni argomentato su questo assunto, per non ripetermi rinvio almeno a Corso di Diritto costituzionale, Padova, 2008, 1 ss.

27 Si veda infra.

28 Fin troppo evidente appare il riferimento al metodo di riflessione professato da C. Schmitt nel notissimo saggio dal titolo / tre tipi di pensiero giuridico (1934) ora in Id., Le categorie del 'politico', cit., 245 ss. su cui torneremo a proposito della definizione del positivismo ottocentesco di matrice legalistica. 
29 Anticipando, in parte, le nostre conclusioni, con riferimento al diritto costituzionale contemporaneo il substrato politico non si trova semplicemente dietro, a monte della costituzione positiva, trovandosi ormai dentro di essa, in quanto appunto positivizzato.

30 C. Schmitt, I tre tipi di pensiero giuridico, cit., 266 ss., indica quale "carattere peculiare" del positivismo "il legame fra normativismo e decisionismo", osservando come "il positivismo...si sottomette alla decisione del legislatore che si trova in possesso del potere statale". In questa prospettiva è esemplare l'impostazione critica di P. Grossi, Società, diritto, stato. Un recupero per il diritto, Milano, 2006, XI, 10 s., 51 s., 100 ss., 112 ss., 116 s.; Id., L'Europa del diritto, Roma-Bari, 2007, 15, 111 ss., 134 ss., 140 ss., 174 ss., 199, 248 ss. che si spinge però fino a travolgere le stesse radici del costituzionalismo. Ma si veda l'approfondita riflessione di G. Zagrebelsky, La legge e la sua giustizia, cit., spec. 110 ss., 114 s.

C. Schmitt., op. ult.cit., 270 s. Si vedano, ancora, N. Bobbio, II positivismo giuridico, cit., 27 ss., 117 ss., 130; A. Pace, Metodi interpretativi, cit., 36 s.; Id., Art. 21, cit., 8 s.; Id., Interpretazione costituzionale, cit., 14 s. ;A. Baldassarre, Miseria, cit., 202 ss., 209 ss.; G.U. Rescigno, Interpretazione costituzionale, cit., 38 ss., 40 ss.

32 A. Baldassarre, Costituzione e teoria, cit., 640 ss., con riferimento anche alla parallela elaborazione teorica portata avanti, per l'Italia, da Vittorio Emanuele Orlando. Sul punto si sofferma anche F. Spantigati, L'acqua è bagnata, cit., pti 22 s. II tema era stato peraltro già oggetto di una approfondita e colta riflessione da parte di G. Azzariti, Dalla discrezionalità al potere, Padova, 1989. Dello stesso autore si veda, altresì, La "prima» scuola italiana di diritto pubblico tra continuità e rotture, già in Pol.dir. 1997, ora in Id., Forme e soggetti della democrazia pluralista, Torino, 2000, 19 ss., spec. 28 s. Lo studio delle questioni connesse alla nascita della scienza italiana del diritto pubblico, qui soltanto accennate, non può oggi prescindere, infine, dal fondamentale contributo di A. Sandulli, Costruire lo Stato. La scienza del diritto amministrativo in Italia (1800-1945), Milano, 2009, spec. 1 ss., 49 ss.

33 Ritengo di poter rilevare un concreto esempio di tale prospettiva di metodo nel saggio di G-U. Rescigno, L'atto normativo, Bologna, 1998.

34 Che se non fraintendo è, in estrema sintesi, anche la prospettiva teorica in cui si muove S. Civitarese Matteucci, Miseria del positivismo giuridico?, cit., spec. 708 ss.

35 Ogni rinvio al pensiero di studiosi di scienze sociali in un'ottica di accostamento reciproco tra gli impianti metodici e concettuali in temini di orientamento culturale alla ricerca di linee di sviluppo di scuole di pensiero omogenee è tendenzialmente incauto, fortemente a rischio di clamorosi equivoci se non arbitrario. Dalla lettura di alcuni recenti lavori non posso però non ricavare alcuni caratteri comuni, nel senso indicato nel testo. Mi riferisco, ad esempio, ai saggi di F. Schauer, Le regole del gioco. Un'analisi filosofica delle decisioni prese secondo le regole nel diritto e nella vita quotidiana, Oxford, 1991, tr.it. a cura di C. Rustici, Bologna, 2000; J.L. Coleman, La pratica dei principi. In difesa di un approccio pragmatista alla teoria dl diritto, Oxford, 2001, tr.it. di V. Alabiso, a cura di G. Pino, Bologna, 2006 ; C. Luzzati, La politica della legalita. II ruolo del giurista nell'età contemporanea, Bologna, 2005 ; S. Civitarese Matteucci, La forma presa sul serio. Formalismo pratico, azione amministrativa e illegalità utile, Torino, 2006, spec. 50 ss.; Id., Miseria del positivismo giuridico?, cit., 686 ss., 708 ss..

A. Pace, Art. 21, cit., 1 ss. Per un'esemplare declinazione in concreto di questa prospettiva di metodo si veda il notissimo saggio La causa della rigidità costituzionale. Una rilettura di Bryce, dello Statuto albertino e di qualche altra costituzione, $2^{\mathrm{a}}$ ed., Padova, 1996, ora anche in Id., Potere costituente, rigidità costituzionale, autovincoli legislativi, $2^{\mathrm{a}}$ ed., Padova, 2002, 1 ss., di cui si segnala altresì la Introduzione, VII ss.

37 A. Baldassarre, Costituzione e teoria, cit., 650, esprime questa sintesi con l'efficace formula: 'La teoria di Kelsen è un monumento giuridico alla democrazia". Si veda, altresì, quanto osserva S. Civitarese Matteucci, La forma presa sul serio, cit., 34. Sul punto trovo esemplare la riflessione di N. Irti, II salvagente della forma, Roma-Bari, 2007, V ss., anche se essa andrebbe approfondita criticamente inserendola nella più generale valutazione dei rapporti tra formalismo e nichilismo giuridico nel pensiero dell'illustre studioso. Si veda, infatti, già N. Irti, Nihilismo giuridico, Roma-Bari, 2004, spec. 18 ss.

38 F. Sorrentino, Le fonti del diritto italiano, Padova, 2009, 1 ss.

39 G. Zagrebelsky, La legge e la sua giustizia, cit., 124 ss., 131 ss. Lascio per ora impregiudicata la questione del se in tale processo debba parlarsi di positivizzazione di un impianto valoriale di matrice giusnaturalistica, cosa che tra l'altro implicherebbe la necessità di qualificare il tipo di giusnaturalismo professato. Ma su ciò infra.

40 M. Fioravanti, Fine o metamorfosi?, in P. Rossi (a cura di), Fine del diritto?, Bologna, 2009, 57 ss. ha di recente osservato come sia "stupefacente quanto poco si sia compreso, nella storiografia, nelle scienze giuridiche e sociali, nella cultura politica, nelle consapevolezze comuni e diffuse, la portata di questa svolta", l'emanazione delle nuove costituzioni democratiche del secondo Dopoguerra, a partire dal "controllo di costituzionalità derivante dalla nuova supremazia della Costituizone".

41 A. Pace, Art. 21, cit., 4, 8 s.

42 Op.ult.cit., 1, 3, dovendo l'interprete sempre e comunque presumere "la prescrittività del testo costituzionale", ibidem, 4. In altra sede l'illustre autore utilizza la emblematica formula del "metodo 
giuspositivista «temperato»... "o "critico» o «metodo scientifico» tout court" in un contesto di necessario pluralismo culturale, Metodi interpretativi, cit., 38 ss., 41, 60. Si veda, altresì, Id., Interpretazione costituzionale, cit., $14 \mathrm{ss}$. Sull'importanza dello studio della storia per lo scienziato sociale si vedano le riflessioni di A.A. Cenvati, Studio del diritto costituzionale, cultura e impegno etico dei giuristi, in Studi Ferrara, cit., I, 702 ss. Ma si veda, anche, quanto osservato da G. Ferrara, Diritto soggettivo, diritto oggettivo, cit., 17.

(

Potrei qui riassumere la complessa questione come problema della ridefinizione del paradigma culturale kelseniano nel quadro del contesto storico più recente, successivo all'avvento delle costituzioni rigide, lunghe e garantite del secondo Dopoguerra. Ma rinvio, oltre agli studi di M.A. Cattaneo, Positivismo giuridico, cit., 319 ss.; F. Modugno, Positivismo giuridico, cit., 450 ss.; N. Bobbio, II positivismo giuridico, cit., spec. 205 ss.; Id., Hans Kelsen (1973), ora in Id. Dalla struttura alla funzione. Nuovi studi di teoria del diritto, Roma-Bari, 2007, 160 ss.; A. Baldassarre, Costituzione e teoria dei valori, cit., locc. citt.; G. Zagrebelsky, II diritto mite, cit., spec. 39 ss., 127 ss., S. Civitarese Matteucci, Miseria del positivismo giuridico?, cit., locc.citt. e G.U. Rescigno, Interpretazione costituzionale e positivismo giuridico, cit., locc. citt., alle riflessioni di N. Matteucci, Positivismo giuridico e costituzionalismo, in Riv. trim. dir.proc. civ., 1963, 985 ss., 1014 ss., 1042 ss., 1057 ss.; S. Pozzolo, Neocostituzionalismo e positivismo giuridico, Torino, 2001, 5 ss., 85 ss., 142 ss.; A. Schiavello, V. Velluzzi, II positivismo giuridico contemporaneo. Una antologia, Torino, 2005, in particolare il saggio di A. Schiavello, Introduzione. Giuspositivismo o giuspositivismi? Un bilancio sul positivismo giuridico degli ultimi venti anni, ivi, 207 ss., seppur in contesti teorici del tutto differenti. Si veda, inoltre, l'illuminante saggio di G. Azzariti, Diritto e conflitti. Lezioni di diritto costituzionale, in corso di pubblicazione per il tipi di Laterza, spec. cap. 6, compresa l'ultima nota dell'ultimo paragrafo.

$$
\text { sagg }
$$
Formalismo giuridico, in Enc. Dir., XVII, 1968, 571 ss.

Miseria del positivismo giuridico, cit., 208 ss.

Almeno non automaticamente.

Ma si veda anche quanto riferito da N. Bobbio, /l positivismo giuridico, cit., 34 ss., e da P. Grossi, L'Europa del diritto, cit., 160 ss., sulla nota polemica tra Savigny e Thibaut, tra la Scuola storica del diritto ed il movimento per la codificazione.

Rinvio ancora ai lavori di Gaetano Azzariti e Aldo Sandulli citati supra alla nota 28).

Carl Schmitt, un nazista senza coraggio, $2^{\text {a }}$ ed., Firenze, 1999, I, 667 s. Si veda, però, già C. Roehrssen, Il ritorno all'ontologia nel costituzionalismo di Weimar, in Id., Diritto e politica. Lo stentato affermarsi dello Stato borghese in Germania visto con gli occhi dei giuristi, Torino, 1995, spec. 140 s. Sul punto rinvio a quanto a suo tempo ossenvato nel mio La crisi dell'ordinamento giuridico dello Stato rappresentativo, Padova, 2000, spec. 286 ss.

52 Secondo questo stesso approccio critico, così mi pare di intendere, M. La Torre, 'Finché la società sarà questa", cit., osserva che addirittura Zagrebelsky, "escludendo che giustizia e diritto abbiano un fondamento normativo trascendente rispetto alla positività dei fatti o della «cultura», quali che questi siano, e separando nettamente giustizia e valori dalla norma giuridica, assume un atteggiamento teorico ed occupa una posizione giusfilosofica che è quella tipica del giuspositivismo".

53 Sul decisionismo di Schmitt rinvio all'approfondita analisi critica di G. Azzariti, Critica della democrazia identitaria, Roma-Bari, 2005, 26 ss.

54 Proprio in questo esempio si palesa la profonda incoerenza delle scelte di metodo professate qualora queste siano meramente strumentali al regime vigente. Lo stesso Schmitt, come è noto, in altro contesto si professerà profondamente critico verso la teoria dei valori paradossalmente proprio in ragione della contrarietà di questa al positivismo, al normativismo accentuato ed alla legalità, anche a fronte dell'alto tasso di soggettivismo implicato dalla sua declinazione in concreto, così nei due saggi dal titolo $L a$ tirannia dei valori, pubblicati in tr.it. in Rass. dir.pubbl., 1970, spec. 9 ss., 18 ss.

55 Si veda sul punto la riflessione di B. Rüthers, Ideologie und Recht im Systemwechsel, München, 1992 , spec. 90 ss.

56 Op.ult. cit., 56 s., con espresso riferimento al nazismo ed all'epurazione dei giuristi ebrei, compreso Hans Kelsen

57 G. Zagrebelsky, La legge e la sua giustizia, cit., 102 ss., giustamente si interroga circa la natura di «stati di diritto» delle "dittature, dei totalitarismi... del nazismo e del fascismo".

58 In ciò, comprendo, 'fl giuspositivismo (diviene) la variante giuridica dell'autoritarismo politico", come sostenuto da A. Baldassarre, Miseria, cit., 208, 213. Quanto alla torsione della legalità in strumento dell'autoritarismo rinvio, per non ripetemi, al mio La legalità: il tramonto di una categoria giuspolitica, in www. costituzionalismo. it, 3/2008, 12 luglio 2008. 
Così il positivismo avrebbe imposto di prestare acriticamente ossequio alla legalità prodotta nelle forme delle leggi razziali, trattando il materiale giuridico "per come esso è'! Si vedano, in proposito, i saggi dedicati a quel "diritto positivo" nel bel volume L. Garlati, T. Vettor (a cura di), II diritto di fronte all'infamia del diritto. A 70 anni dalle leggi razziali, Milano, 2009, nonché il saggio di P. Caretti e A. Cardone, La parabola della legislazione razziale. Alcuni appunti in tema di fonti del diritto, in Scritti in onore di Lorenza Carlassare, a cura di G. Brunelli, A. Pugiotto, P. Veronesi, V, Napoli, 2009, 2213 ss. E' in fondo piena adesione alla dottrina positivista anche la declinazione della linea di difesa dei criminali nazisti ispirata dalla pretesa di avere soltanto obbedito alle leggi. Non a caso G. Rossi, I/ gioco delle regole, Milano, 2006, 98 proprio con riferimento a questi episodi parla di crisi del positivismo "sconfitto sul campo dai regimi antidemocratici e dai sistemi giuridici a base razziale". Così, ancora, A. Pace, Le sfide del costituzionalismo nel XXI secolo, ora in Id., I limiti del potere, cit., 5.

61 Ma sulle contraddizioni del pensiero giuspolitico weimariano e con specifico riferimento al pensiero di Heller si vedano, almeno, le riflessioni di M. La Torre, La crisi del Novecento. Giuristi e filosofi nel crepuscolo di Weimar, Bari, 2006, spec. 46 ss., 97 ss., 113 ss.; e di C. De Fiores, Masse, nazione $e$ nazionalizzazione delle masse nella teoria giuridica fascista, in Scritti Carlassare, cit., V, 2258 ss., spec. 2269 ss., 2292 ss..

62 E' evidente qui il riferimento alla linea di sviluppo tracciata fin dal suo sottotitolo dal saggio di G. Ferrara, La Costituzione. Dal pensiero politico alla norma giuridica, cit.

63 A. Baldassarre, Costituzione e teoria dei valori, cit., 650. Ma sui limiti della teoria kelseniana della Costituzione e proprio nella prospettiva qui seguita si veda anche quanto osservato da G. Ferrara, La Costituzione, cit., 233 ss.; Id., Sulle fonti del diritto. Qualche premessa, in www.costituzionalismo.it, 2/2006, 19 maggio 2006, spec. §§XXIX e XXX, laddove denuncia i rischi che la teoria di Kelsen possa implicare l'affermazione di un'idea del diritto "opposta alla ragione e alla storia del costituzionalismo e a favore di chi detiene il potere, comunque lo abbia acquisito. Infatti, non risponde la teoria di Kelsen alla domanda più spontanea che possa mai essere posta a questo riguardo: perché bisogna ubbidire alle norme, a queste norme? La verità è che la tesi della legittimazione di un ordinamento basata sulla autoreferenzialità 'della produzione di norme a mezzo di norme' occulta il reale fondamento di ogni ordinamento giuridico, fondamento che si evince dalla constatazione della sua effettività".

64 A volte si ha quasi l'impressione di dover lottare contro l'imposizione di una sorta di neoaristotelismo scientifico seppur non di matrice religiosa, che in ossequio ad una tradizione culturale ed alla conseguente elaborazione teorica si ritiene debba prevalere anche contro l'evidenza dei dati empirici.

65 Per un'approfondita analisi critica del normativismo quale matrice della "composizione procedurale dei conflitti” G. Azzariti, Diritto e conflitti, cit., cap. 6.

66 L. Ferrajoli, Principia iuris, I, 847 ss.

67 Rinvio ancora a quanto più sopra osservato in relazione alla parabola storica del positivismo giuridico.

68 "Il suo retroterra storico-politico e socio-culturale...è garanzia di «neutralità» e di «avalutatività» proprio perché si astiene dal considerare i fini, supponendoli per dati”, A. Baldassarre, Miseria, cit., 206, corsivo mio; G. Ferrara, Sulle fonti del diritto, cit., locc. citt.

69 Op.ult.cit., 208.

70 'La congiunzione essenziale fra autoritarismo politico e positivismo giuridico ha dominato la Staatslehre dell'era bismarckiana", A. Baldassarre, op. ult. cit., 213.

71 Sulle specifiche critiche sul punto formulate al pensiero di Kelsen da H. Heller si vedano le interessanti riflessioni di M. La Torre, La crisi del Novecento, cit., spec. 97 ss.

72 G. Ferrara, Sulle fonti, cit., § XXIX.

73 II diritto pubblico verso la "teoria generale»: G. Jellinek, in Diritto e politica, cit., 82 ss.

74 A. Baldassarre, op. ult. cit., 220.

75 Op.loc.ult.cit.

76 Si veda la prospettiva di riflessione di cui ai recenti saggi di G. Zagrebelsky, La legge e la sua giustizia, cit., 205 ss.; Id., Intorno alla legge, cit., spec. 85 ss., 102 ss.; G. Silvestri, Dal potere ai principi. Libertà ed eguaglianza nel costituzionalismo contemporaneo, Roma-Bari, 2009, 3 ss. Ma già C. Pinelli, II dibattito sull'interpretazione costituzionale fra teoria e giurisprudenza, in Scritti in memoria di Livio Paladin, III, Napoli, 2004, 1665 ss.

77 Cosi ancora A. Baldassarre, op.ult.cit., 221.

78 Specialmente in regime maggioritario, laddove il diritto di rango legislativo, la legalità ordinaria possono divenire funzione dell'arbitrio del potere di governo, quindi diritto "di parte", 'legalità separata", nel senso da me già denunciato nello scritto La legalità: il tramonto di una categoria giuspolitica, cit. Si veda, altresì, quanto osservato da M. La Torre, 'Finchè la società sarà questa', cit., 20 del dattiloscritto.

79 Trovo esemplare, sul punto, la riflessione di B. Celano, Giusnaturalismo, positivismo giuridico $e$ pluralismo etico, in Materiali per una storia della cultura giuridica, 2005, 161 ss., che appunto imposta la sua analisi sul dato per cui "principi e valori etici sono necessariamente (salvo ipotesi di scuola) parte del diritto", 165.

80 G.U Rescigno, Interpretazione costituzionale e positivismo giuridico, cit., 43 ss. 
Qui mi limito a poche citazioni formali: tra i tanti, ad es., U. Scarpelli, II positivismo giuridico rivisitato, ora in A. Schiavello, V. Velluzzi, II positivismo giuridico contemporaneo, cit., 104 ss.; A. Schiavello, Introduzione. La crisi del positivismo giuridico, ivi, 117 ss.; S. Pozzolo, Neocostituzionalsimo, cit., 5 ss.; L. Ferrajoli, Principia iuris, cit., I, 848 s., 902 s.; Id., II, Teoria della democrazia, 29 ss.40 ss.; M. La Torre, 'Finché la società sarà questa', cit., $2 \mathrm{~s}$.

82 Sono, qui, fortemente debitore dell'impianto di metodo professato in G. Ferrara, I/ diritto come storia, in Dir.pubbl., 2005, 1 ss.

83 P. Ridola, II costituzionalismo: itinerari storici e percorsi concettuali, in Studi Ferrara, cit., III, 316 ss.

84 Rechtsstaat im wandel, Stuttgart, 1964, tr.it. a cura di C. Amirante, Milano, 1973.

85 Basti rileggere le pagine del saggio del 1959 La trasformazione della legge costituzionale, ivi, 195 ss.

86 Op.ult.cit., 198, corsivo mio.

87 Op.ult. cit., 202.

88 "q'allontanamento dal formalismo della costituzione" che ne usi le disposizioni secondo il paradigma del "concetto di legge dello stato di diritto", op.ult.cit., 220.

89 Op.ult.cit., 216.

90 La "perdita dei caratteri formali del diritto costituzionale è lo sviluppo dello stato di giustizia, che si manifesta soprattutto nell'istituzione della giurisdizione costituzionale", op. ult.cit., 229, corsivo mio.

91 Op.ult.cit., 230 s.

92 M.G. Losano, Prefazione, cit., VI s.; M. La Torre, Filosofia e scienza giuridica, cit., 10 ss., 15 s., 24 ss. parla di vero e proprio "salto", di "svolta". Ma sul punto lo stesso Bobbio, Dalla struttura alla funzione, cit., 184.

93 Milano, 1977, ora ripubblicati, come già ricordato, con una Prefazione di M.G. Losano, Roma-Bari, 2007, XIX s., 48 ss., 67 ss., 75 ss., 184 s..

94 Sul punto, ampliamente S. D'Albergo, Diritto e stato tra scienza giuridica e marxismo, Roma, 2004, spec. 272 ss. a proposito della necessità di abbandonare l'idea stessa di democrazia come metodo formale di costruzione delle regole del gioco verso un'idea di democrazia sociale partecipata, quella professata dalla Costituzione italiana, appunto. Dopo Weimar, anche la Costituzione italiana, nel funzionalizzare la proprietà privata, 'traccia le condizioni di legittimità di un «uso» della legge....in vista del «fine da raggiungere»...la tipologia dei fini è formalmente prevista dalla Costituzione", 283. Per una concreta applicazione del paradigma si veda, ora, il bel saggio dedicato a Silvio Trentin da F. Cortese, Libertà individuale e organizzazione pubblica in Silvio Trentin, Milano, 2008, spec. 64 ss., 249 ss.

95 Come è evidente, ad esempio, in N. Bobbio, op.ult.cit., $184 \mathrm{~s}$. Ma sul punto, espressamente, M. La Torre, Filosofia e scienza giuridica, cit., $27 \mathrm{~s}$.

96 'Una concezione propriamente normativa della costituzione non può essere formulata che in senso funzionale (...) il profilo funzionale della costituzione è irrinunciabile", M Dogliani, Costituzione in senso formale, materiale, strutturale e funzionale: a proposito di una riflessione di Gunther Teubner sulle tendenze autodistruttive dei sistemi sociali, in www. costituzionalismo.it, 3/2009, 17 novembre 2009.

97 In questo senso, questa mi pare la prospettiva della riflessione condotta da G. Azzariti, Diritto e conflitti, cit., Parte seconda. Le citazioni nel testo sono tratte dal par. 2. Ve precisato, peraltro, che Azzariti critica radicalmente le dottrine funzionaliste del diritto ( $\$ 3.1$ del cap. 5 della Parte seconda) con ciò, però, intendendo riferirsi non alla funzione della costituzione quale strumento complessivo di composizione o di legittimazione dei conflitti, bensì alla lettura strumentale, tecnocratica, formalista dell'ordinamento e delle sue procedure per la soluzione di singole, specifiche controversie. Questa lettura rischia infatti di compromettere la dimensione costituzionale - in senso sostanziale - dell'ordinamento, nella opposta attitudine a legittimare la riduzione della complessità sociale, la semplificazione della problematica dei conflitti attraverso una costante soluzione tecnica di ciascun singola controversia in luogo della gestione della complessità sociale, producendo nei fatti una selettiva stabilizzazione di una categoria di esclusi. Ma per ogni specifico approfondimento di tale critica non possiamo che rinviare al saggio citato.

98 Qui non posso che rinviare all'illuminante saggio di C. Roehrssen, I/ diritto pubblico verso la "teoria generale», cit., 67 ss.

99 Op.ult.cit., 78. 'Valga il vero; quando in una situazione sociale completamente mutata il giurista comincia a dare un peso diverso alla cultura politica sottostante alla costruzione giuridica (è il caso di Carl Schmitt) evidentemente cessa di fare il giurista di un certo tipo ed incomincia a svolgere una funzione che al giurista di quel tipo sarebbe sembrata politica sans phrase", 79.

100 Ciò in coerenza con le riflessioni a suo tempo svolte nel mio La crisi dell'ordinamento giuridico, cit., Parte seconda, cap. IV dal titolo 'Riflessioni sul declino delle categorie del sistema giuridico", 283 ss. a proposito della crisi della validità, del sistema quale categoria essenziale dello stesso fenomeno giuridico, del normativismo, del positivismo quale categoria ordinante del diritto: quindi della stessa idea di "sistema delle fonti".

$101 \mathrm{Ne}$ parla S. D'Albergo, Dalla democrazia sociale alla democrazia costituzionale (un percorso dell'ideologia giuridica, in www.costituzionalsimo.it, 3/2005, 14 ottobre 2005. 
102 Modello stigmatizzato, tanto per fare alcuni esempi, dal recente volumetto di L. Vandelli, Psicopatologia delle riforme quotidiane. Le turbe delle istituzioni: sintomi, diagnosi e terapie, Bologna, 2006, ma anche, già quasi dieci anni prima da M. Ainis, La legge oscura. Come e perché non funziona, Roma-Bari, 1997.

103 Basti il rinvio al recente studio di C. Pinelli, Un sistema parallelo. Decreti-legge e ordinanze d'urgenza nell'esperienza italiana, Relazione al Convegno del Gruppo San Martino su 'Recenti novità nell'uso dei poteri normativi del governo", Università di Milano Bicocca, 13 novembre 2009, in www.associazionedeicostituzionalisti. it.

104 Sulla crisi dei rapporti tra scienza giuspubblicistica e legislatore positivo rinvio a quanto a suo tempo scritto nel volume su La crisi, cit., 286 ss., anche in riferimento alle riflessioni critiche formulate da J.H. von Kirchmann, Über die Vertlosigkeit der Jurisprudenz als Wissentschaft (1848), tr.it. di P. Frezza, La mancanza di valore della giurisprudenza come scienza, 1942 e, a partire da questo stesso saggio, da C. Schmitt, La condizione della scienza giuridica europea (1943-1944), tr.it. di A. Carrino, 1996.

105 B. Celano, Giusnaturalismo, positivismo, cit., 176 ss.

106 'Il diritto è una specifica tecnica di bilanciamento, determinazione, commisurazione, risoluzione di conflitti pratici: è un sistema dinamico", op.loc.ult.cit.

107 Per la declinazione di tale prospettiva di riflessione rinvio di nuovo al saggio di G. Azzariti, Diritto $e$ conflitti, cit., cap. 7.

108 F. Rimoli, Pluralismo e valori costituzionali. I paradossi dell'integrazione democratica, Torino, 1999, 200, 213 ss., assumendosi la costituzione il compito di garantire "...il dominio del mutamento...la costituzione pluralistica si pone come...luogo di ricomposizione, nelle forme del procedimento, del conflitto e di semplificazione della complessità nel momento integrativo".

109 L'interprete della Costituzione di fronte al rapporto fatto-valore, cit., 21. La Costituzione "contiene regole che aspirano a quella che potremmo chiamare l'eternità ordina mentale", 22.

110 Se non fraintendo è questo, ad esempio, l'esito dell'articolata e colta riflessione di F. Rimoli, Pluralismo $e$ valori, cit., spec. 255 ss. Nella prospettiva indicata trovo inoltre sintomatiche le tesi formulate nel Manifesto di giuristi per un Ritorno al diritto: i valori della convivenza quale programma di politica culturale della omonima Rivista, 2004, 15 ss., ispirato dall'intento di porre al centro della riflessione dei giuristi "non la norma, ma l'apprezzamento della situazione e dei valori" per un 'ritorno al diritto quale valutazione dei valori coinvolti nella convivenza", $16 \mathrm{~s}$. su cui Una opinione dissenziente di G. Azzariti, ivi, 98 ss. criticamente proprio sul progetto di "dare valutazione giuridica" non più "secondo il diritto positivo", ma "secondo i valori della convivenza". Ma si veda, altresì, F. Spantigati, L'acqua è bagnata, cit., pti 44 ss. del testo dattiloscritto.

111 Emergenza, interpretazione per valori e certezza del diritto, cit.

112 Sull'analisi del diritto come scienza pratica e per una critica dei "procedimenti logici rigorosi, a carattere deduttivo" resta esemplare la ricerca filosofica di Alessandro Giuliani, sui cui si veda ora il bel saggio di F- Cerrone, Alessandro Giuliani: un'idea di ragione critica, dialettica e controversiale per il diritto, in www. dircost. unito. it dizionario.

113 Così, ad esempio, A. Baldassarre, Interpretazione e argomentazione, cit., 28.

114 Lo Stato di diritto: un'introduzione storica, in P. Costa, D. Zolo (a cura di), Lo Stato di diritto. Storia, teoria, critica, Milano, 2002, spec. 191 ss.

115 Introduzione allo studio del diritto costituzionale. Le basi del costituzionalismo inglese (1885), 1915, tr.it della versione inglese del 1959, rist. London-New York, 1964, a cura di A. Torre, Bologna, 2003.

116 Op.ult.cit., 193.

117 Intorno a questa categoria avevo già riflettuto nel mio precedente lavoro La legge e l'interesse generale: un paradigma per un'etica costituzionale?, in L. Chieffi (a cura di), Rappresentanza politica, gruppi di pressione, élites al potere, Torino, 2006, 313 ss., spec. 326 ss.

118 Op.ult.cit., $198 \mathrm{~s}$.

119 Devo questa conclusione ad una conversazione con Stefano Civitarese.

120 Cit. Sul confronto di metodo prodotto dal concorso del neocostituzionalismo alla denunciata crisi di effettività del positivismo giuridico si veda anche l'interessante studio di V. Omaggio, Teorie dell'interpretazione. Giuspositivismo, ermeneutica giuridica, neocostituzionalismo, Napoli, 2003, spec. 93 ss.

121 Ritengo utile segnalare che M. La Torre, 'Finché la società sarà questa', cit., ascriva alle correnti neocostituzionalistiche sia Zagrebelsky che Ferrajoli, seppur considerando soltanto quest'ultimo di ascendenze analitiche, $14 \mathrm{s.}, 16,18 \mathrm{~s}$.

122 Del resto a questo sembrano fare riferimento le citate riflessioni di B. Celano, Giusnaturalismo, positivismo, cit. Non a caso M. Barberis, Sette domande a Bruno Celano, in Materiali per una storia, cit., 2005, 185 ss. insiste proprio sul valore polisenso e sulla confusione di ruoli che, nel pensiero di Celano, si producono intorno alle nozioni di giuspositivismo e di giusnaturalismo.

123 Così espressamente A. Pace, Art. 21, cit., 5; Id., Interpretazione costituzionale, cit., 16, 20. Ma si veda già quanto osservato in Id., Metodi interpretativi, cit., 42, 52 ss., 60 s.; Id., Le sfide del costituzionalismo, 
cit., 11 ss. Rischi che M. Luciani, Costituzionalismo irenico, cit., 14 ss, rinviene altresì nelle dottrine del c.d. "costituzionalismo multilivello".

124 A. Pace, Le sfide, cit, 12 ss.17 ss.; G. Azzaniti, Interpretazione e teoria dei valori: tornare alla Costituzione, in A. Palazzo (a cura di), L'interpretazione della legge alle soglie del XXI secolo, Napoli, 2001, 231 ss.; G.U. Rescigno, Interpretazione costituzionale, cit., 44 ss.; G. Ferrara, Diritto soggettivo, diritto oggettivo, cit., $17 \mathrm{ss}$.; M. Luciani, L'interprete della Costituzione di fronte al rapporto fatto-valore. II testo costituzionale nella sua dimensione diacronica, in Dir.Soc., 2009, spec. 18 ss.

125 A. Pace, Art. 21, cit., 6.

126 Richiamerei qui quasi alla lettera i contenuti dei saggi, ivi pubblicati, di G. Ferrara, Le ragioni di una rivista nuova, 5 febbraio 2003; G. Azzariti, Le ragioni di un impegno nuovo, 9 luglio 2003; Id., Per un diritto costituzionale "normativo", n. 1/2004, 27 ottobre 2004; M. Dogliani, Validità e normatività delle costituzioni (a proposito del programma di Costituzionalismo.it), n. 2/2004, 18 gennaio 2005, ora anche in Studi Ferrara, cit., II, 243 ss.

127 Posizione che, credo, almeno in quest'ultimo senso, sia condivisa anche da A. Pace, Le sfide del costituzionalismo, cit., 4 ss.; Id., Interpretazione costituzionale, cit., 4, 18.

${ }_{128}$ Espressamente G. Ferrara, Le ragioni, cit. e G. Azzariti, Le ragioni, cit., fin dai relativi incipit

129 M. Dogliani, Validità e normatività, cit., 3.

130 G. Ferrara, op. ult.cit., 3.

131 G. Azzariti, op. ult. cit., 2 s.

132 M. Dogliani, op. ult. cit., 1.

133 A. Pace, Interpretazione costituzionale, cit., 20 che, peraltro, mantiene rigorosamente distinta la prospettiva idelogica del costituzionalismo dal tenore testuale del diritto costituzionale vigente nel dato momento storico. L'assunto riportato nel testo mi sembra, peraltro, condiviso anche da A. Baldassarre, Interpretazione e argomentazione, cit., 1, 8, $20 \mathrm{~s}$.

134 M. Dogliani, op. ult. cit., 2.

135 Art. 21, cit., 10.

136 M. Troper, II concetto di costituzionalismo e la moderna teoria del diritto, in Materiali per una storia della cultura giuridica, 1988, 61 ss; L. Ferrajoli, Principia iuris, cit., 1., cit., 26 ss., 2., 31 ss. 41 ss. Si rinvia, inoltre, alla riflessione sul punto di G.U. Rescigno, Interpretazione costituzionale, cit., 40 ss.

\section{Recebido para publicação 12/11/2009}

Aceito para publicação 13/12/2009 